\title{
SÉLECTION SÉMANTIQUE ET SÉLECTION NATURELLE \\ LE Rôle CAUSAL du LEXIQUe
}

Certains systèmes physiques - nous en l'occurrence - ont la curieuse propriété de pouvoir agir, et agir très spécifiquement, en fonction de ce qu'on leur dit. Il est parfaitement légitime, j'entends légitime du point de vue épistémologique, qu'on explique le pourquoi et le comment d'une action en remontant à un ordre verbal, ou écrit. En d'autres termes, un énoncé peut être la cause d'un certain comportement. Lorsque, selon notre psychologie spontanée, nous croyons bon d'expliquer une action par ce qui a été dit, nous présupposons dejà un certain déterminisme et une certaine finesse de grain de cette causalité. Malgré sa parfaite appartenance à l'ordre naturel, ce lien est ancré sur des structures abstraites hautement complexes et on n'a pas la moindre idée de la façon dont on peut l'analyser en des termes et des concepts propres aux sciences physiques. Dans cet article, je me propose de développer quelques considérations élémentaires sur la nature du lien causal entre actions et énoncés linguistiques, et en particulier sur le rôle fondamental que le lexique d'une langue naturelle joue dans cette causalité.

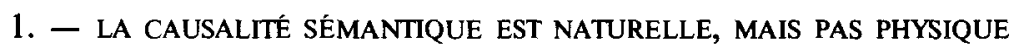

A seule fin d'aider notre intuition, analysons, par exemple, les enchầnements d'actions spécifiques et distinctes, tous prévisibles, qui peuvent suivre ces différents ordres :

(1) Il faut le persuader qu'il doit partir.

(2) Il faut le persuader de partir.

(3) Il faut le faire partir.

Il nous est évident que (3), à l'opposé de (1) et (2), permet l'utilisation de menaces et chantages, tandis que (2) (un peu à la limite) et (3), mais pas (1), admettent la possibilité d'acheter carrément le départ par une 
certaine somme. Il est aussi évident que cette différence s'explique en grande partie par la différence de signification entre les expressions "persuader" et "faire partir». Ce lien d'une grande finesse entre actions et ordres, actions et suggestions, ou mème actions et questions bien visées, fait partie de notre existence quotidienne. C'est bien sa "banalité " qui fait problème, du point de vue scientifique, puisque l'évidence même de ce lien démontre que nous sommes en présence d'un processus mental routinier, en grande partie inconscient, et, comme nous le verrons, de nature largement déductive.

Un autre aspect central de cette causalité est sa vaste indépendance par rapport au contexte. La spécificité des comportements qui peuvent suivre un ordre comme (1) ou (3) est pour nous généralement sans mystère, même quand on ne sait ni de qui il est question, ni quelles raisons motivent le locuteur. Il est clair, par exemple, en dehors de toute connaissance du contexte, que le locuteur de (1), (2) et (3) n'est pas la personne qui doit être persuadée, ou induite, à partir. Si tel était « l'état de choses " que le locuteur avait voulu exprimer ou qu'il avait voulu voir réalisé, il se serait plutôt exprimé ainsi :

(4) Il faut me persuader de partir.

La différence entre les actions qui suivent (4) et celles qui suivent (2) s'explique entièrement par la difference entre la contribution des mots "le" et "me" à la signification de chacun de ces énoncés. Il faut souligner aussi que la finesse de ce lien causal ne peut pas s'expliquer autrement. Cette causalité, indépendante par rapport au contexte, qui relie les énoncés aux actions, je l'appellerai, en m'inspirant des travaux de James T. Higginbotham (Higginbotham, 1985, 1986, 1988)*, une causalité sémantique. Causalité morpho-syntactico-sémantique serait peut-être un meilleur terme, mais il est trop lourd, et je préfère l'appeler simplement sémantique, après avoir dûment souligné au départ son indépendance par rapport au contexte. Je me propose, dans cet article, de montrer qu'il s'agit d'une causalité parfaitement naturelle. Mais il y a un certain intérêt épistémologique à souligner qu'elle ne se laisse pas réduire à une causalité physique.

En effet, les sciences physiques, telles que nous les concevons actuellement, n'ont aucun moyen de saisir les fondements mêmes de cette causalité. Par exemple, l'énergie acoustique véhiculée par les sons correspondants est totalement incapable d'expliquer le phénomène qui nous intéresse ici. L'univers des phénomènes sémantiques est étranger à l'activation sélective d'un système par l'intermédiaire d'une " touche " vocale.

\footnotetext{
* Pour plus de précisions concernant les références placées entre parenthèses dans cet article, se reporter à la Bibliographie, p. 90.
} 
Il y a activation sélective, mais grâce à la médiation inéliminable de schémas abstraits, et non par l'effet d'une transduction directe des ondes de pression. A la différence de certaines machines dites " intelligentes" qui sont activées directement par la voix humaine, c'est-à-dire par les propriétés physiques de paquets d'ondes acoustiques, nos actions et nos pensées sont sélectivement engendrées par des structures abstraites que nous construisons sur la base des stimuli acoustiques. Nous projetons activement sur les paquets d'ondes acoustiques une famille de structures abstraites sous-jacentes, en correspondance étroite les unes avec les autres à plusieurs niveaux ${ }^{1}$. La causalité qui relie les expressions linguistiques à nos actions et à nos pensées doit faire appel à cette famille de structures abstraites et à leurs principes de correspondance ponctuelle, principes qui sont, comme nous le verrons par la suite, en partie universels et en partie spécifiques à une langue donnée. La structure physique du stimulus acoustique n'est certainement pas «simple", mais elle est néanmoins immensément plus pauvre que ce que notre appareil linguistique nous livre à la fin du processus de projection. Le résultat final du système de projections est bien ce qu'il nous faut pour fonder le processus de sélection sémantique. Parmi toutes les significations humainement concevables, une et une seule (parfois deux, et plus rarement encore trois ou quatre, dans le cas des expressions fortement ambiguës) sera choisie comme la signification de cette expression. Cette sélection est quelque chose que chaque être humain, dès son âge le plus tendre, fait normalement, sans effort, sans incertitude et presque instantanément. Il s'agit donc d'une sélection "naturelle ", mais à ne pas confondre avec le processus évolutionniste darwinien qui porte le même nom. Les raisons de cette distinction apparaitront plus clairement par la suite.

Il est important de souligner ici que les prétendues analogies que certains zoo-sémioticiens ont voulu établir entre le langage humain et la communication animale sont trompeuses. La nature abstraite, ouverte, infinie, compositionnelle, créative et générative des phénomènes sémantiques qui nous intéressent ici échappe à toute modélisation fondée sur un répertoire fini, discret et clos de commandes et de signalisations (voir les commentaires de plusieurs auteurs à Daniel C. Dennett, in Dennett, 1983). Comme l'a souligné Noam Chomsky (Chomsky, 1955, in Chomsky, 1985), les nombres naturels et les langues naturelles constituent les deux seuls systèmes " naturels " et biologiquement accessibles qui sont à la fois discrets et infinis. Aucune autre espèce animale ne peut

1. D'ordinaire ces niveaux reçoivent en grammaire générative les noms respectifs de : Forme Phonologique, Structure D, Structure de Surface et Forme Logique (CHOMSKY, 1981, 1986 ; MAY, 1985 ; RiemSDIJK \& WilliamS, 1986 ; LASNIK \& URIAGEREKA, 1988). 
se servir de systèmes possédant ces deux attributs à la fois, puisque toute communication animale est fondée, ou bien sur un répertoire discret et fini (de cris, de gestes, etc.), ou bien sur un canal à modulation continue d'intensité (phéromones, pirouettes, attouchements, etc.). La nature compositionnelle, abstraite, discrète et infinie de nos langues exclut que l'on puisse expliquer la sélection sémantique par une liste finie et figée de correspondances entre énoncés et significations. Il ne s'agit pas de trouver la touche intérieure (la signification) qui serait actionnée par la touche extérieure (le son) et qui, à son tour, déclencherait un comportement ou une représentation mentale. Il est fondamental de comprendre que la causalité sémantique ne se laisse reconduire à aucun schéma de ce genre. Même le lexique, comme nous le verrons par la suite, est un répertoire de structures abstraites de haute complexité, un ensemble fortement structuré et richement articulé, aussi bien au niveau global qu'au niveau de ses composants individuels. Un ensemble qui, de surcroît, demeure toujours "ouvert" à de nouvelles insertions (Richard J. Carter, 1984 a).

Rappelons aussi qu'il est impossible de saisir le phénomène de la causalité sémantique par des corrélations statistiques. Il est bien connu, par exemple, que les correspondances privilégiées (ou même obligées) à longue distance entre les termes d'un même énoncé défient le calcul des probabilités ${ }^{2}$. Qui plus est, la projection des structures syntaxiques semble engendrer obligatoirement des éléments "vides " (empty categories) qui sont cruciaux pour déterminer la signification, mais qui ne pourraient être soumis à aucun calcul statistique, pour la simple raison qu'ils ne sont pas présents du tout dans le stimulus manifeste (ils n'ont pas de "réalité phonologique") et leur probabilité de parution est rigoureusement nulle (Chomsky, 1981, 1986; Lasnik \& Uriagereka, 1988 ; Van Riemsdijk \& Williams, 1986).

Un autre argument familier, qui souligne le manque d'intérêt des analyses statistiques en linguistique, est fondé sur un simple calcul combinatoire : n'importe quelle phrase de sept mots aurait une probabilité a priori ridiculement faible d'être présentée au locuteur. Plusieurs siècles peuvent tranquillement s'écouler sans que cet énoncé soit jamais présenté, même si une nouvelle phrase de sept mots était produite chaque seconde. D'où l'idéalisation courante en linguistique, selon

2. Il est évident à tous les locuteurs du français que dans la phrase : « Anne s'est demandé si c'était bien Jean qui a eu l'idée saugrenue d'interdire strictement à Ginette d'aller la voir à l'hôpital ", il y a une forte présomption de co-référence entre "Anne " et "la ", malgré le fait que ces deux mots sont séparés par 21 autres mots. Le calcul des probabilités ne parviendrait jamais à rendre compte de ces corrélations à longue distance (CHOMSKY, 1955; maintenant in CHOMSKY, 1985). 
laquelle chaque énoncé a toujours la même probabilité a priori d'être présenté : la probabilité zéro (Chomsky, 1985; Elman, 1989; MarslenWilson \& Tyler, 1980 ; Salasoo \& Pisoni, 1985). Cette idéalisation élimine, entre autres, tout traitement de la sémantique basé sur la théorie classique de l'information, au sens de Shannon et Weaver. Il s'ensuivrait, en effet, qu'un énoncé quelconque, ayant une probabilité a priori égale à zéro, serait porteur d'une quantité d'information littéralement infinie. Il ne s'agit pas d'un paradoxe intéressant, mais d'une banale réduction à l'absurde, obtenue en appliquant un modèle très pauvre à un domaine qui n'est point de sa pertinence. La sémantique des langues naturelles est, tout simplement, étrangère à la théorie de l'information.

Souligner, comme je viens de le faire, que la causalité sémantique n'appartient pas au domaine des sciences physiques, ni à celui de la statistique, est parfaitement compatible avec la thèse que cette causalité appartient entièrement au monde naturel et que l'on peut, en principe, en trouver une explication scientifique. Sur la base de quelques contributions récentes (en partie encore non publiées), et sans prétendre en fournir ici une revue exhaustive, je voudrais présenter un schéma de solution naturaliste et non réductionniste qui me semble acceptable. La théorie que je peux à peine esquisser ici est une théorie naturaliste, compositionnelle et fortement innéiste des contenus sémantiques et des processus par lesquels ceux-ci peuvent être la cause aussi bien de comportements manifestes que d'états mentaux strictement "privés $"^{3}$. En ce qui concerne le lexique, et donc les concepts " primitifs" (ou, plus exactement, les concepts qui sont mono-morphémiquement lexicalisables), ma théorie est atomiste, mais dans un sens un peu particulier : chaque signification lexicale est un individu doue d'une structure interne très riche et très spécifique. Chacun de ces atomes de la langue connecte causalement le locuteur de cette langue avec une certaine portion du monde, ou avec un certain état des choses, qui nous sont accessibles sous une certaine description. La causalité sémantique est une causalité de dicto et non pas seulement de re. Un point capital sur lequel nous reviendrons par la suite.

3. Il est sous-entendu que cette causalité présuppose toujours une certaine situation standard : que le message acoustique ou graphique ne soit pas trop perturbè par des facteurs de bruit, que le sujet soit physiquement, physiologiquement et psychologiquement capable d'entendre et d'agir, que ses compétences linguistiques et pragmatiques soient conformes aux normes courantes. Tout ce que je dirai ici fait tacitement appel à des conditions banales de ceteris paribus (FODOR, 1987, 1989). 


\section{2. - LA TENTATION BÉHAVIORISTE}

Sous peine de circularité, une théorie causale naturaliste des contenus sémantiques doit agencer les éléments des langues naturelles (par exemple, le mot 'chat') avec des états de faits " publics » (l'animal de ce nom), sans présupposer déjà la nature foncièrement sémantique du lien. Une solution classique, celle de Skinner, consistait à dire que la présence physique d'un chat dans le voisinage du locuteur, sous certaines conditions normales d'illumination, d'attention, d'élaboration visuelle, etc., rend fort probable que ce locuteur prononce audiblement le son linguistique 'chat' (ou un son équivalent dans les diverses langues naturelles). L'idée centrale de cette approche bien connue est que la probabilité $a$ priori d'entendre l'expression 'chat' est nettement plus grande lorsqu'il y a ou lorsqu'il y a eu quelques minutes auparavant, un chat aux alentours du locuteur. Une corrélation objective entre deux fréquences objectives (manifestations publiques d'objets et énonciations audibles de sons), tel est le fondement de cette sémantique béhavioriste.

Il devint vite clair, toutefois, que cette approche s'engouffrait dans des contrefactuels du type : si $x$ avait été présent, au lieu d'y, alors on aurait observé $\mathrm{X}$, au lieu de $\mathrm{Y}$, avec probabilité Px. A bien y regarder, la " force " de ces raisonnements par contrefactuels se fondait toujours sur des relations nomologiques qui présupposaient la nature sémantique du lien et qui étaient, en conséquence, incapables de fonder ce lien. Un petit sommaire des déboires de la sémantique skinnerienne nous sera utile.

Il y a tout de même un petit point en faveur de la théorie béhavioriste, comme le souligne Jerry A. Fodor dans un manuscrit récent (Fodor, 1989). La théorie est, en effet, parfaitement " atomiste », car la connexion sémantique skinnerienne entre la bête et le son 'chat' pourrait tout aussi bien se matérialiser chez une espèce idéalisée qui ne possède, en tout et pour tout, qu'un seul concept : le concept 'chat'. En outre, même chez une espèce comme la nôtre, qui possède une énorme variété de concepts, le mécanisme causal qui relie Félix au concept 'chat' est, de facto et en principe, indépendant du mécanisme qui relie Médor au concept 'chien'. On a là deux mécanismes de même nature mais, dans l'univers des connexions causales entre concepts et objets, chacun de ces liens reste indépendant de tous les autres, aussi bien psychologiquement que, pour ainsi dire, métaphysiquement. Pour ceux qui, comme Fodor et moi, apprécient le caractère atomiste d'une théorie sémantique, cette indépen- 
dance est un aspect positif. Malheureusement, bien d'autres aspects de la théorie skinnerienne ne le sont pas du tout.

Noam Chomsky a montré il y a longtemps (Chomsky, 1959) que la caractérisation de la situation "normale " de présentation de l'objet au locuteur se révèle être déjà foncièrement intentionnelle, c'est-à-dire, qu'elle présuppose tacitement la nature sémantique du procès qu'elle était censée expliquer. En bref, car l'histoire est trop bien connue pour qu'on s'y attarde, ce qui compte comme condition " normale " de présentation suivie par vocalisation ne se laisse nullement réduire à une liste " innocente " de situations physiques. Par exemple, le sujet prononcera le mot 'chat' si, et seulement si, il pense que quelqu'un aux alentours l'écoutera et sera intéressé à entendre l'expression 'chat'. Donc, la définition primaire de la normalité de la situation ne peut pas faire l'économie d'une composante mentaliste, qui est par nécessité bâtie sur des éléments qui sont déjà sémantiques. En outre, la théorie est totalement incapable d'etablir une distinction entre les vocalisations (et donc les concepts) 'chat', 'chat-ou-lapin', 'félin', 'chat-avant-le-coucher-dusoleil', ' animal-qui-chasse-les-souris', 'animal-préféré-par-ma-grandmère' et une infinité d'autres concepts dont la bête présentée ici et maintenant constitue aussi un exemplaire. N'importe quelle propriété qui co-varie causalement avec la présence de cet animal peut en fin de compte être, dans le schéma de Skinner, un candidat acceptable par la signification du mot 'chat'. Que dire, en outre, de la présentation d'un "stimulus" comme «la deuxième guerre mondiale"?

Dans ce rappel sommaire des failles de la théorie béhavioriste, il faut au moins mentionner le problème de la disjonction et le problème des associations mentales (Dennett, 1987, 1988; Dretske, 1981, 1986, 1988 ; Fodor, 1987, 1989 ; Loar, 1981 ; Ruth Millikan, 1984, 1986, 1989; Segal \& Sober, 1989). Un gros lapin que l'on entrevoit de loin par une nuit sans lune peut aussi causer la vocalisation 'chat', mais la signification de 'chat' n'est pas 'chat ou lapin-par-une-nuit-sans-lune'. La théorie sémantique béhavioriste n'a jamais pu résoudre ce problème redoutable et il y a de bonnes raisons de croire qu'elle ne pourrait pas le résoudre par ses propres moyens ultra-minimalistes et anti-mentalistes. L'autre problème insoluble pour la théorie béhavioriste est qu'une souris en plein jour peut aussi, par une association d'idées qui est parfaitement "normale", causer la vocalisation 'chat'. Comme Fodor le souligne, le problème de la disjonction et le problème des chaînes d'associations mentales guettent non seulement la théorie skinnerienne, mais toutes les approches naturalistes proposées jusqu'ici. Il nous faut introduire d'autres critères naturalistes, capables enfin d'expliquer pourquoi 'chat' est un concept primitif, tandis que 'chat ou lapin-par-une-nuit-sans-lune' est 
un concept dérivé. En l'absence de contraintes spécifiques sur ce qui peut compter comme concept primitif, sur ce qui va être fixé en premier et de préférence lorsque nous sommes actuellement en présence d'un exemplaire de cette espèce, nous ne pourrions jamais décider si 'chat' veut dire 'chat' ou plutôt 'chat ou lapin-par-une-nuit-sans-lune'.

Un début d'explication nous vient des sciences cognitives (Carey, 1985 ; Keil, 1979 ; Markman, 1989 ; Smith \& Medin, 1981 ; Pinker, 1989) et de la sémantique lexicale (Baker, 1988 ; Dowty, 1979; Fillmore, 1968 ; Fodor, 1987; Grimshaw, 1979; Hale \& Keyser, 1989; Higginbotham, 1986; Jackendoff, 1983, 1987; Lederer, Gleitman \& Gleitman, 1989 ; Levin \& Tenny, 1988; Macnamara, 1982; Pinker, 1989; Pustejovsky, 1988; Rappaport \& Levin, 1988; Talmy, 1985; Tanenhaus, Garnsey Boland, 1989; Tenny, 1988 a, 1988b). L'acquisition des concepts par l'enfant est contrainte par une classe de concepts « naturels ", qui sont toujours appris en priorité et sur la base desquels les autres concepts dérivés (notamment les concepts disjonctifs) sont successivement construits. Comme nous le verrons par la suite à propos de l'acquisition du lexique, il y a des significations (chat, par exemple) qui sont obligatoirement exprimées par un seul mot dans n'importe quelle langue naturelle, tandis que d'autres significations doivent être exprimées par des périphrases. Pour reprendre un exemple bien connu de Quine (Quine, 1960), mais avec des conclusions radicalement opposées à celles qu'il en tire, aucune langue humaine possible ne peut avoir un seul mot pour exprimer 'partie-de-lapin-non-détachée' et cinq mots pour exprimer 'lapin'4.

Quine, qui se prétend skinnerien, croyait nous avoir appris à vivre paisiblement avec le problème de la disjonction, car il croyait nous avoir démontré qu'il n'y a, en principe, aucune façon de le trancher. Selon Quine, le choix entre 'chat', 'chat-ou-lapin', 'partie-de-chat-nondétachée', etc. reste à jamais indécidable, car il est dicté uniquement par des critères de simplicité pratique, par une convention tacite ayant une

4. Dans la fameuse situation imaginaire de Quine, un anthropologue-linguiste voit un lapin dans un pré et entend un indigène prononcer le mot 'gavagai' en indiquant la bête. Quine soutient qu'il est impossible de décider en principe si la traduction correcte de l'expression 'gavagai' est 'lapin' ou 'partie-de-lapin-non-détachée', ou 'lapinitéexemplifiée-ici-et-maintenant', ou une infinité d'autres expressions de ce type. Si 'gavagai' est un seul mot de la langue de l'indigène, et si on n'a le choix qu'entre les trois traductions qui précèdent, alors, sans même y réfléchir, on sait que 'gavagai' doit signifier 'lapin'. Cette connaissance est une connaissance innée, tacite et modulaire. Il n'y a aucune nécessité logique dans cela, mais il se trouve que notre système langagier est ainsi bâti. Les contraintes sur les significations possibles de nos lexiques naturels ne proviennent pas de la logique, mais de notre constitution biologique. Il se trouve que le choix entre 'lapin' et 'partie-delapin-non-détachée ' comme candidats à la signification du son 'gavagai' ne se pose même pas, ni à l'adulte, ni à l'enfant. Le problème de la disjonction est résolu au préalable par notre système langagier, c'est-à-dire, indirectement, par notre constitution spécifique. 
certaine utilité sociale et par un principe de " charité " sémantique. Selon Quine, il est simplement sage et prudent de ne pas attribuer à autrui, sans y être contraint, un appareil sémantique trop différent du nôtre. Il s'agit là d'une bonne règle de parcimonie saine et utile, mais qui laisse le problème de la disjonction et de «l'indétermination de la traduction " entièrement ouvert.

La théorie sémantique de Quine est naturaliste, mais, à la différence de celle de Skinner, c'est une théorie globale (ou « holiste »), car le système des symboles joue comme un tout, chaque symbole recevant sa valence sémantique par sa position au sein du réseau de tous les autres termes de la langue. L'atomisme de Skinner est abandonné, en dépit d'une prétendue allégeance quinienne à la stricte doctrine béhavioriste. Ce que Quine nous offre c'est une théorie holiste et fortement indéterministe. Quine admet, toutefois, des contraintes biologiques, liées à l'évolution de notre espèce. En effet, ce qui unit plus profondément la sémantique de Quine et celle de Skinner, et les unit ensemble à beaucoup d'autres sémantiques naturalistes, c'est ce que j'appelle la tentation darwinienne. Encore une tentation à laquelle il faut résister.

\section{3. - LA TENTATION DARWINIENNE EN SÉMANTIQUE}

Tout semble se résoudre, même le problème de la disjonction, si l'on suppose que les "mauvaises " connexions sémantiques entre le monde et les significations ont été éliminées par les simples mécanismes de la survie. Depuis l'aube de ce qu'on appelle aujourd'hui l'épistémologie évolutionniste, c'est-à-dire depuis Charles Sanders Peirce (1896), Ludwig Boltzmann (1904), Konrad Lorenz (1941), jusqu'à nos jours, avec les théories sémantiques de Ruth Garreth Millikan (1984, 1986, 1989), Fred Dretske (1981, 1986, 1988), Daniel C. Dennett (1987, 1988), en passant par Karl R. Popper (1972), Donald T. Campbell (1974) et Willard Van Orman Quine (1974), cette solution a été retenue comme inévitable et parfaitement satisfaisante. Le lien offert par l'explication sélective darwinienne est bien un lien causal, car la démographie réelle des individus serait rigoureusement causalement covariante avec la démographie abstraite des contenus sémantiques. A la base de cette explication se trouve le raisonnement contrefactuel suivant : si nos mots et nos concepts avaient été mal « connectés » avec le monde, nous ne pourrions pas être ici pour en discuter, parce que nos ancêtres auraient été dévorés par les bêtes féroces ou anéantis dans des catastrophes naturelles. Puisque nous 
sommes toujours là, il s'ensuit que notre sémantique "colle avec" le monde, du moins en première et bonne approximation. Ce que j'appelle ici la sélection sémantique serait, selon cette théorie, un effet direct de la sélection naturelle darwinienne.

Cette approche ne tombe pas dans la circularité de vouloir expliquer le sémantique par le sémantique, car, dans l'univers darwinien, on survit, ou on disparait, objectivement, sans même savoir pourquoi, en fonction des concepts que l'on acquiert. Il ne peut, en outre, y avoir de lien plus "naturaliste", puisqu'il s'agit du même lien qui relie n'importe quelle structure biologique à ses fonctions, dans le cadre écologique "normal " de chaque espèce.

La théorie sémantique évolutionniste semble donc solide, causale, naturaliste et, si l'on attache de l'importance à ce point, aussi atomiste. En effet, les mésaventures sélectives par lesquelles les ancêtres des chats actuels ont fixé dans les esprits de nos ancêtres le concept 'chat' peuvent certainement être, en principe, causalement indépendantes des mésaventures par lesquelles les rochers de l'époque ont fini par fixer le concept 'rocher'. Comme il se doit, dans une théorie atomiste, deux mécanismes peuvent être de même nature sans pour autant dépendre causalement l'un de l'autre.

Les difficultés exorbitantes que rencontre toute théorie évolutionniste darwinienne des contenus mentaux ont fait l'objet d'un exposé détaillé (Piattelli-Palmarini, 1988 b). Je me bornerai ici à en esquisser quelques aspects particulièrement pertinents pour mon propos.

Même en admettant que la théorie sémantique darwinienne puisse devenir aussi solide qu'il le faut, c'est-à-dire qu'elle puisse parvenir à corréler les objets du monde et les concepts d'une manière suffisamment forte et à l'épreuve des contrefactuels, elle ne sera jamais assez fine, elle n'arrivera jamais à être assez spécifique. Selon l'expression de Fodor, la causalité des mésaventures darwiniennes est insensible aux descriptions sous lesquelles ces mésaventures nous sont arrivées (ou plutôt, sont arrivées à nos ancêtres). En termes de survie différentielle, il est indifférent d'être dévoré par un tigre, par le seul félin qui possède des rayures sur fond jaune, par le seul mammifère qui possède la séquence génétique ...CCATTGG..., ou par l'animal que ma grand-mère déteste le plus. Mais les descriptions sous lesquelles les choses nous sont mentalement présentes, et sous lesquelles nous nous rendons compte de ce qui nous arrive, sont essentielles à la sémantique. Un être doué de raison, même d'une toute petite raison, ou d'une proto-raison, doit pouvoir se rendre compte de ce qui lui arrive et des raisons pour lesquelles cela lui arrive. Notre capacité de décrire, de présenter à autrui et de nous représenter mentalement, que $\mathrm{x}$ est un $\mathrm{X}$, et non pas un $\mathrm{Y}$, est bien indispensable à la 
constitution même du tout premier embryon de sémantique. Pourtant, selon les mécanismes de la survie différentielle darwinienne, peu importe que la description de l'animal qui nous dévore soit $\mathrm{X}$ ou $\mathrm{Y}$, et que la description sous laquelle nous sommes dévorés soit $Z$ ou $W$. Ces mécanismes de survie darwinienne, bien connus (et utilisés de façon largement abusive), se moquent de la sémantique, parce que seul compte pour eux le nombre objectif des descendants et des disparus, en corrélation avec les causes génétiques et écologiques objectives. Le schéma darwinien n'arrive pas à engendrer une causalité de dicto, car il est seulement sensible à une causalité de re. Mais une causalité de re est foncièrement insuffisante à fonder une sémantique.

La théorie darwinienne ne peut pas sortir d'une double impasse en ce qui concerne la sémantique : si elle devient causalement dépendante des descriptions subjectives, elle perd sa force objective et elle présuppose déjà ce qu'elle était censée expliquer; si, par contre, elle ne descend pas à ce niveau, elle reste à jamais étrangère à l'univers des significations. Ce qui donne à la théorie toute sa force (le compte objectif des cadavres et des survivants) lui soustrait du même coup la capacité d'engendrer une sémantique. Il me semble clair, donc, que les contraintes adaptatives dictées par la survie peuvent constituer, au mieux, une vague toile de fond sur laquelle la causalité sémantique reste encore entièrement à esquisser.

Les mécanismes sémantiques que nous possédons (comme bien d'autres structures et fonctions) sont, certes, compatibles avec un récit évolutionniste darwinien, mais ils restent radicalement sous-déterminés par celui-ci. Il y a une infinité de systèmes sémantiques possibles, qui seraient tout aussi compatibles avec les contraintes darwiniennes, mais qui ne sont pas les nôtres ${ }^{5}$. Seules les limites de notre imagination nous interdisent de concevoir en détail une infinité de systèmes sémantiques qui seraient

5. Par exemple, imaginons des êtres fort semblables à nous, mais chez lesquels tous les termes qui décrivent des objets solides font référence uniquement à la surface de ces objets. Doués d'un pareil système, nos ancêtres auraient parfaitement réussi à échapper aux tigres, à éviter de se cogner contre les rochers et à cueillir les fruits des arbres, mais il leur serait apparu " évident " que, par exemple, l'estomac du tigre n'est pas une partie de l'animal, mais quelque chose qui se trouve « près du tigre ", tandis que les rayures font partie du tigre, car elles se trouvent sur la surface. Cette sémantique engendrerait, probablement, deux termes et deux "concepts " distincts pour la tortue (un lorsque la tête et les pattes sont dehors, un autre lorsqu'elles sont à l'intérieur). Des créatures raisonnables équipées avec un pareil système de représentations mentales se feraient, probablement, une idée un peu spéciale, que sais-je?, des mystères du sexe et de la procréation (le fotus serait, pour eux, 'près de la mère', et j'en passe). Pourtant, rien de cela ne rendrait ces êtres imaginaires, doués d'un système si radicalement différent du nôtre, incapables de survivre et de se multiplier. Tous les cas de significations authentiquement « impossibles " pour nous (voir, par exemple, les travaux de Richard J. Carter) seraient parfaitement compatibles avec la survie darwinienne. 
pour nous tout à fait " innaturels", voire « inhumains ", mais parfaitement compatibles avec les critères darwiniens de survie.

En sémantique, il faut donc résister à la tentation darwinienne, car il nous faut des contraintes naturelles immensément plus fines. Les contraintes qui peuvent commencer à fournir cette explication sont presque certainement de lointaine origine biologique, mais elles sont radicalement sous-déterminées par la simple survie différentielle darwinienne (Piattelli-Palmarini, 1989).

Ayant écarté malentendus et faux-départs, je me propose maintenant d'esquisser la nature des principales contraintes naturelles en sémantique.

\section{4. - LES CONTRAINTES LEXICALES}

Le caractère compositionnel de la sémantique de nos langues naturelles (Fodor \& Pylyshyn, 1988; Higginbotham 1986; Carter, 1984 a, b) suggère que l'on commence par les unités élémentaires, c'est-à-dire par les termes individuels du lexique. Les contraintes naturelles sur les significations lexicales possibles seront très importantes pour nous. Sur ce point, toutefois, il nous reste encore à nous libérer de certains malentendus. La subtilité et la richesse des lexiques de nos langues nous ont joué un mauvais tour, car elles nous ont fait croire que n'importe quel concept, concret ou abstrait, simple ou complexe, individuel ou agrégé, ancien ou moderne, est potentiellement exprimable par un mot unique. Or une étude comparative de la structure universelle du lexique, en liaison étroite avec celle de la syntaxe et de la morphologie, a révélé de fortes contraintes sur ce qui est "nommable " (plus exactement, monomorphémiquement lexicalisable). Il ne s'agit nullement de contraintes agissant sur, ou dictées par, les limites de notre intelligence. Ces contraintes sont de nature exclusivement linguistique. Par exemple, il y a une infinité de choses que nous pouvons aisément exprimer par une circonlocution, mais que nous ne pouvons exprimer par un seul mot. Supposons que le verbe imaginaire 'fersuader' signifie " persuader une femme" et prenons un énoncé aussi simple que le suivant :

(1) Jean n'est pas capable de fersuader.

Nous ne savons pas si ce qui est nié par (1) est la capacité à persuader les femmes, ou à persuader tout court, ou les deux à la fois. Il nous est 
difficile de décider ce que (1) veut dire. Le concept n'est certainement pas trop " difficile " pour notre entendement, pourtant nos stratégies naturelles, irréfléchies, de dérivation sémantique se trouvent bloquées. « Fersuader " n'est pas un concept mono-morphémiquement lexicalisable. Disons, par simplicité, que ce n'est pas un verbe possible d'une langue naturelle. Les choses deviendraient, en effet, très vite compliquées, si on considérait des énoncés tels que :

(2) Personne ne peut fersuader n'importe comment.

(3) N'importe qui peut être fersuadé par quelqu'un.

(4) Quelqu'un peut fersuader sans aucun effort.

Nous sommes sortis des limites naturelles d'élaboration et de sélection sémantique; les procédés spontanés, irréfléchis qui nous guident sans aucune peine, instantanément, en présence des termes ordinaires de nos lexiques naturels ne peuvent plus nous aider ${ }^{6}$.

D'autres exemples de mots (et de significations mono-lexicales) impossibles seraient : 'panger' (manger du pain et...) et 'blire' (lire la bible, mais pas...). Dès que l'on essaye des phrases qui nient, ou conditionnent hypothétiquement ces verbes imaginaires, ou opèrent sur eux avec des quantificateurs, on est perdu. La formation de simples mots composés, comme 'immangeable', 'illisible' (ou comme les équivalents de 'déboutonner', 'surcharger', etc.), se trouve aussi bloquée. Si l'on essaye ces constructions avec des verbes impossibles, on obtient des termes dont la signification nous échappe totalement. Par exemple, l'anglais admet par sa morphologie la combinaison de suffixes de comparaison et de superlativisation (-er, -est) avec des préfixes de négation (un-, in-, counter-) (comme dans unhappier, unkindlier, unpleasanter, etc.). La compréhension de la porté (scope) logique de ces modifications, pourtant si routinières, se trouverait totalement bafouée dans le cas de verbes impossibles (Pesetsky, 1985). Il nous faut réfléchir longuement avant de décider ce qui $a$ été dit. Malgré ces efforts exceptionnels, il n'est pas sûr que nous puissions toujours y arriver ${ }^{7}$. Par contre, la négation et la quantification

6. Par exemple, quelle est la valeur de vérité de (2) s'il y a au moins un homme qui peut $p$ ersuader n'importe comment n'importe qui ? Ou celle de (3) si, pour chaque femme, il y a toujours une autre femme, mais aucun homme, qui peut la $f$ ersuader? Que dire de (4), s'il se trouve que tous ceux qui peuvent persuader sans effort n'ont jamais essayé avec aucune femme? Il nous faut quelques instants de réflexion avant de répondre, peut-être même un papier et un crayon.

7. Un exemple : « Aucun livre n'est assez banal pour ne pas être blu » (rappel : 'blire' = ' lire la bible, mais pas...'). Que faire d'une expression pareille? 
des termes des langues naturelles n'occasionnent jamais des difficultés de ce genre. Celles-ci montrent bien qu'il s'agit de contraintes spécifiquement lexicales, et non pas cognitives, ou computationnelles génériques. Il est, en effet, facile de trouver des périphrases qui peuvent exprimer aisément, et précisément, ces significations " impossibles", des périphrases que l'on découvre être d'une parfaite banalité conceptuelle.

Tout ceci témoigne que des termes imaginaires comme 'fersuader', 'blire' et 'panger' ne pourraient jamais devenir les termes d'une langue naturelle possible. Aucun enfant ne parviendrait à les "découvrir " dans une situation ordinaire (j'exclus ici la situation où l'on propose à l'enfant une devinette amusante).

Nous voyons donc qu'il y a des limites à ce qu'un seul mot peut vouloir dire dans une langue naturelle quelconque. Nous touchons là aux contraintes incontournables de notre système linguistique. Ces contraintes sont bien incontournables, quoique parfaitement contingentes, liées à notre biologie, et non pas à la logique pure, ou à l'efficacité de la communication. Pour les raisons analysées il y a un instant, ces contraintes ne proviennent pas non plus de mécanismes de la survie darwinienne.

Sur la base de considérations beaucoup plus techniques, que je ne peux résumer ici, il est apparu que ces contraintes sont parfaitement, quoique tacitement, accessibles à l'enfant lorsqu'il apprend sa langue maternelle. Une infinité d'hypothèses bizarres (comme 'fersuader', 'panger', 'blire', etc.), pourtant toutes compatibles avec les données visuelles ou tactiles disponibles, ne seront jamais envisagées par l'enfant. Non parce que l'expérience les contredit, mais parce que ce ne sont pas des hypothèses sémantiques humainement accessibles. Un extra-terrestre ou un ordinateur pourrait fort bien les envisager, mais pas un être humain. L'enfant ne supposera jamais que 'persuader' puisse faire référence uniquement à un des deux sexes ${ }^{8}$. Pourtant, il y a des termes du lexique qui se réfèrent à un seul sexe : sœur, maritorne, soubrette, feee, etc., mais aussi robe/costume, bas/chaussettes, etc. Cette subtilité du lexique, ainsi que la richesse des mots et des significations, défient le caractère, pourtant, si évident de ces multiples contraintes. Historiquement, cette difficulté nous a conduit à conclure qu'il n'y avait pas de contraintes du tout et que tout ce qui était pensable était aussi monolexiquement " effable".

Il y a, au contraire, des contraintes sémantiques lexicales nettes et puissantes, qui sont pour nous si profondément évidentes et spontanées

8. Ou que 'chaussure' puisse signifier uniquement chaussure gauche, même si ce qu'on lui montre est une chaussure gauche. 
que les linguistes arrivent à les dépasser seulement par un effort soutenu de l'imagination. Dans ce domaine, les généralisations scientifiques solides ne sont pas simples et les dernières années nous ont appris que la comparaison en profondeur de beaucoup de langues, couplée avec une analyse fine des effets syntaxiques de ces contraintes lexicales sont indispensables au progrès (Tenny, $1988 \mathrm{a}$ ).

Actuellement, les deux principales voies d'accès aux contraintes lexicales sont constituées par certaines conséquences sémantiques déductibles des contraintes morphologiques et syntaxiques, et par des cas hypothétiques de significations impossibles (tels que celui que je viens d'envisager) (Hale \& Keyser, 1988, 1989 ; Jackendoff 1983, 1987 ; Pustejovsky, 1988 ; Tenny, 1988 a) ${ }^{9}$. La recherche sur la sémantique lexicale est en plein essor et il est difficile d'en anticiper les résultats. Je peux seulement en résumer quelques traits saillants. Il ne s'agit, pour l'instant, que de vérifier quelques-uns des types de contraintes agissant à notre insu sur le lexique de nos langues.

\section{5. - UN CAS IMAGINAIRE : L'ACQUISITION DU VERBE « PLONCHER »}

Le cas idéalisé que je veux développer ici est celui de l'enfant qui rencontre un mot, donc une signification lexicale, ou un 'concept', pour la première fois. Sur la base des travaux de James T. Higginbotham $(1985,1986,1988)$, je veux reconstruire certaines contraintes indépendantes du contexte, et par là réfléchir sur l'enchevêtrement étroit de la morphologie, de la syntaxe et de la sémantique ${ }^{10}$.

9. Une vraie floraison de significations lexicales impossibles a été brillamment engendrée et minutieusement examinée par Richard J. Carter au long des années (voir le recueil de ses écrits, paru en 1988 sous la direction de Beth Levin et Carol Tenny). Les exemples de Carter portent aussi sur la comparaison entre différentes langues et touchent à beaucoup de problèmes fondamentaux en morphologie, en syntaxe, en sémantique et aussi en philosophie du langage (un certain nombre de ces articles ont été publiés initialement en français; voir, par exemple, CARTER, 1984c; CARTER, 1980).

10. Des auteurs tels que Ray Jackendoff et Leonard Talmy font aussi intervenir des contraintes objectives, spatio-temporelles et " actantielles ", sur les significations possibles. Certaines contraintes perceptuelles et conceptuelles générales, dictées par les structures topologiques de l'espace, par l'enchainement des événements dans le temps, et par les types de mouvements possibles (ou concevables), seraient reflétées, selon eux, dans les significations lexicales. Cette approche a été formalisée mathématiquement (et aussi radicalisée quant à sa portée ontologique) par l'école « catastrophiste " française. René Thom et Jean Petitot tendent à donner à ces contraintes sur les significations un statut objectif formel, de nature intrinsèquement morphodynamique. Les structures sèmantiques des langues naturelles 
Je considère comme acquis que chaque énoncé manifeste est associé à une structure riche et spécifique, qui n'est que partiellement exprimée au niveau phonétique, mais qui est mentalement et tacitement représentée en tous ses détails à plusieurs niveaux d'abstraction (Chomsky, 1981; Lasnik \& Uriagereka, 1988; Riemsdijk \& Williams, 1986). Entre ces différentes représentations, il y a des correspondances ponctuelles obligées, qui ne sont pas accessibles consciemment au sujet (Chomsky, 1981, 1986 ; Lasnik \& Uriagereka, 1988; May, 1985 ; Pesetsky, 1985, 1987 ; Riemsdijk \& Williams, 1986). Ces structures cachées exercent des contraintes spécifiques et puissantes sur la signification du mot inconnu (ou imparfaitement connu, mais je veux ici analyser le cas le plus difficile). Pour rendre la situation intuitivement plus saisissante, je vais utiliser des mots inventés. Ce petit stratagème innocent nous permet de mieux nous « identifier » avec l'enfant.

Dans une situation que l'on peut aisément imaginer, l'enfant demande à sa maman ce qu'elle est en train de faire, et il entend la réponse suivante :

(1) Maman est en train de ploncher ce poisson.

Nous dirions que l'enfant n'a « aucune idée » de ce que ce verbe (imaginaire dans mon cas, mais réel dans la situation que je veux reconstruire) peut vouloir dire. Nous le dirions, mais nous avons tort, car la structure syntaxique de (1) véhicule déjà un bon nombre de contraintes sémantiques. Le fait qu'elles soient évidentes et banales n'empêche pas qu'elles soient capitales. "Ploncher" est quelque chose que maman (sujet) fait au poisson (objet) ${ }^{11}$. Il s'agit d'un verbe transitif (et qui exclut par le fait même d'innombrables significations comme dormir, neiger, flotter, évaporer, etc.). «En train de " signifie que cette action aura par la suite son résultat. Ce que maman a fait n'est pas déjà la «plonchade » ou le " plonchage ", c'est une action partielle dans un schéma plus vaste, qui s'étale dans le temps.

Ces données syntaxiques excluent d'ores et déjà une quantité d'autres

seraient donc plus fondamentales, plus générales, plus profondes et plus révélatrices que leurs conséquences aux niveaux cognitifs, syntaxiques, etc. (PETrTot, 1989, 1985). Je me bornerai ici à souligner que l'extrême spécificité des données, sur lesquelles les linguistes de l'école générative travaillent, reste au-delà de la portée explicative de tels formalismes encore envisagés par Petitot.

11. Comme l'a montré en détail Lila Gleitman, mème le tout jeune enfant déduit des significations différentes, spécifiques et parfaitement prévisibles, à partir d'un même dessin (ou photo), quand on lui décrit l'action d'un verbe imaginaire exemplifié par le dessin (ou la photo) comme : "A plonche B " par opposition à "A et B plonchent" (LEDERER, Glemman \& Gleitman, 1989). 
significations impossibles. Ploncher ne peut pas être un verbe dit d'achèvement inéluctable, un de ces verbes qui caractérisent des actions telles que, lorsqu'on commence à en faire un tout petit peu, on les a déjà achevées (heurter, rencontrer, reconnaître, sursauter, etc.). Ce ne peut, non plus, être un verbe dit « vrai statif » (true stative) (avoir, savoir, aimer, ressembler, etc.), car aucun de ces verbes n'admet la construction « être en train de... $" 12$.

Il faut répéter que beaucoup de renseignements cruciaux sont aussi extraits par l'enfant de son observation visuelle, par exemple des gestes que sa mère effectue sous ses yeux, des outils dont elle se sert, etc. (il est crucial, par exemple, que ploncher est quelque chose que maman peut faire à elle toute seule, avec les outils disponibles dans la maison). Les sciences cognitives nous ont appris combien de contraintes perceptives, conceptuelles et sémantiques spontanées, non apprises, universelles, agissent aussi sur ces composantes perceptuelles, ontologiques et dans les attributions d'intentions à autrui (Carey, 1985 ; Gleitman, 1986; Goldstone, Gentner \& Medin, 1989 ; Jackendoff, 1983; Keil, 1979; Landau \& Gleitman, 1985 ; Markman, 1989 ; Smith \& Medin, 1981; Spelke, 1985, 1988). Mais limitons-nous, pour l'instant, aux contraintes sur la signification qui se manifestent au niveau morpho-syntaxique.

Supposons que maman ajoute :

(2) Regarde chéri comme c'est facile de ploncher ce poisson.

(3) Ce poisson se plonche très facilement.

(4) Ce poisson est très facilement plonchable.

Une quantité d'informations nouvelles devient accessible à l'enfant. Pour nous en rendre compte, il suffit de comparer (4) avec des couples de verbes, pourtant presque synonymes, qui admettent, ou n'admettent pas, la construction-able. Par exemple : 'présenter-présentable', mais pas 'exhiber-*exhibable'; 'naviguer-navigable', mais pas 'ramer-*ramable'; 'surmonter-surmontable', mais pas 'excéder-*excédable'; 'redouterredoutable', mais pas 'craindre-*craignable'; 'connaître-connaissable',

12. Il est intéressant de remarquer que cette construction peut forcer une expression à prendre parfois une signification dynamique, un devenir au lieu d'un être. Par exemple : " Il est en train de ressembler de plus en plus à son père "; "Il est en train d'avoir de plus en plus d'argent ». En l'absence d'une expression qui souligne fortement la progression (comme " de plus en plus »), ces expressions seraient inacceptables (cf. *« Il est en train de savoir la réponse ») ou métaphoriques (« Il est en train d'aimer Marie »). (Je remercie Jean-Michel Roy et François Dell de m'avoir suggéré ces exemples). 
mais pas 'savoir-*sachable'. L'absence de ces termes ne provient pas de considérations logiques, ou cognitives d'ordre général, mais semble être liée à des critères morphosyntaxiques extrêmement subtils ${ }^{13}$.

Il est capital de souligner que, si ce dialogue s'était tenu en anglais, les informations auraient été encore plus riches et plus subtiles. Puisqu'il s'agit de contraintes sémantiques déduites (inconsciemment) de la syntaxe, il ne faut pas s'étonner que la traduction fidèle et exacte d'une phrase de la langue $A$ dans une autre langue $B$ puisse véhiculer davantage d'informations à l'enfant qui parle la langue $B$. En effet, si nous traduisons notre verbe imaginaire "ploncher " par le verbe anglais « to splonk ", tout aussi imaginaire, nous obtenons l'équivalent de (3) :

(3a) This fish splonks easily.

Ceci dira implicitement, mais certainement, à l'enfant de langue anglaise qu'il ne s'agit pas d'un verbe de perception (comme 'see', 'hear', 'sniff', car on ne peut pas dire que quelque chose " ou "*sniffs easily"). En français, par contre, grâce à l'usage du " se ", on peut dire que quelque chose "se voit facilement", ou "s'entend facilement » (Grimshaw, 1982 ; Kayne, 1975). Donc, en dépit de circonstances identiques, l'enfant de langue française qui entend (3) recevra des informations différentes sur ce verbe inconnu que l'enfant de langue anglaise qui entend (3a). Les deux "milieux " ou " situations " sont physiquement et psychologiquement identiques, mais linguistiquement différents. Et ceci est bien ce à quoi il fallait s'attendre, puisque les contraintes sémantiques qui nous intéressent ici sont de nature morphosyntaxique et variables d'une langue à l'autre (pour une analyse étendue voir aussi les travaux de Richard Carter, in Levin \& Tenny, 1988).

Restons encore un instant dans le " milieu » syntaxique de l'anglais. (3a) exclut de nombreuses autres significations (Hale \& Keyser, 1988, 1989): les verbes instrumentaux sans modalité quantifiable en ce qui concerne leur objet (à la différence de ce qui concerne l'instrument luimême), comme 'stab', 'nail', 'hammer', et les verbes dits "vrais statifs ", comme 'know', 'understand', 'trust', car ceux-ci n'admettent pas la construction *... easily ${ }^{14}$. Certains verbes, qui admettent cette construc-

13. Selon certains auteurs, il s'agit d'une structure lexicale projective, déterminée par le fait que le verbe peut ou non "c-commander" son argument interne, et par le nombre de niveaux de dérivation qui séparent la forme de surface et la forme logique (voir l'analyse présentée par PESETSKY, 1985).

14. Il suffit de penser à des expressions syntaxiquement inadmissibles comme : *This plank stabs easily', "This topic knows easily', "John trusts easily' (dans le sens : " Il est facile de faire confiance à John $"$ ), etc. 
tion (dite middle construction en anglais), admettent aussi une construction impersonnelle et sans spécification de l'agent. On peut dire "This glass breaks easily" et on peut, aussi, dire : "This glass broke." Si l'enfant de langue anglaise entend une construction comme celle-ci ("This fish splonked "), il exclut d'emblée, déductivement, beaucoup d'autres significations intéressantes, car la mécanique de la situation pourrait ne pas les exclure par elle-même. Par exemple les actions, ou transformations, suivantes (je les transcris en français pour plus de simplicité): 'saupoudrer', 'hisser', 'couper en tranches', etc.

D'autres contraintes sur la signification possible de 'ploncher' peuvent être tacitement exprimées par des phrases où ce verbe est enchâssé dans des expressions qui partialisent, ou quantifient (measure-out) l'action de 'ploncher' (Tenny, 1988b). Par exemple, il y a une différence entre :

(5) Maman a plonché le poisson à moitié.

(6) Maman a plonché la moitié du poisson.

(7) Maman a mi-plonché le poisson.

On peut aisément, si tant est qu'on le puisse (c'est-à-dire, si la syntaxe du verbe 'ploncher' l'admet), construire d'autres phrases avec 'peu à peu', 'un peu à la fois', 'pendant une heure', 'en deux minutes', 'quasiment', 'presque', 'arrêter de...', etc. (Grimshaw, 1979; Hale \& Keyser, 1989; Jackendoff, 1987; Pustejovsky, 1988; Rappaport \& Levin, 1988 ; Tenny, $1988 \mathrm{a}, \mathrm{b}$ ). Par exemple (5) signale (tacitement, je le répète) qu'il est possible de s'arrêter à mi-chemin dans cette action en ayant, du fait même, déjà fait une "moitié " de l'action, mais seulement une moitié. ('Ploncher' n'est donc pas comme 'frapper', 'secouer', ou 'trébucher', et bien d'autres verbes encore, car ceux-ci ne permettent pas ce genre de "partialisation" progressive). (6) signale qu'un autre type de partialisation est possible : celle qui consiste à compléter l'action, mais sur une fraction d'un seul objet seulement. Si on entend une phrase telle que (6), on peut exclure que 'ploncher' appartienne à la même catégorie que (parmi bien d'autres) 'poignarder', 'tuer', 'ranger', ou 'enfermer' (rappel : 'ce poisson' est un objet individuel, pas une collection d'exemplaires, et pas un amas d'éléments, comme le blé, la farine, le vin, le sable, etc.). (7) signale que l'action est, pour ainsi dire, progressivement mesurable à l'intérieur de son déroulement même, et que 'ploncher' n'appartient donc pas à la même catégorie que 'briser', 'interférer', 'livrer', etc.

Cette analyse pourrait bien s'étendre à d'autres phrases qui qualifient syntaxiquement l'action de 'ploncher' par des quantificateurs adverbiaux ('presque', 'quasiment', 'à nouveau', etc.), par des modalités psycholo- 
giques ('intentionnellement', 'sans vouloir', 'avec tendresse', etc.), par des modalités effectuelles ('discrètement', 'en silence', 'gratuitement', etc.), ou par des temporalisations ('fréquemment', 'en deux minutes', 'pendant une heure', 'en une minute', etc.). La signification possible du verbe s'en trouverait progressivement de plus en plus circonscrite ${ }^{15}$.

Il faut bien souligner que le rythme d'acquisition du lexique par l'enfant, surtout à l'âge de ce qu'on appelle (à juste titre) l'explosion lexicale, est prodigieux : en moyenne, un nouveau mot par heure, pendant des années (Miller, 1986; Miller \& Gildea, 1987). Cette acquisition serait impossible s'il n'y avait pas de multiples contraintes morphosyntaxiques pour délimiter tacitement le nombre de "candidats " admissibles à la signification pour chaque mot nouveau que l'enfant rencontre. Dans un manuscrit en cours de publication, Jacques Mehler et ses collaborateurs montrent que des contraintes strictement phonologiques contribuent puissamment à signaler au tout jeune enfant le début et la fin d'un mot possible de sa langue maternelle. Ces contraintes phonologiques (par exemple, sur la structure des syllabes possibles et sur les positions possibles de l'accent tonique), couplées avec les contraintes morphologiques (Pesetsky, 1985) et les contraintes syntaxiques (Gleitman, 1986; Landau \& Gleitman, 1985; Lederer, Gleitman \& Gleitman, 1989), contribuent aussi à expliquer la rapidité et la finesse de l'acquisition du lexique. La mise en place du système sémantique naturel serait, en principe et en pratique, impossible sans l'aide tacite et puissante de ces principes morpho-syntactico-sémantiques, qui sont inconsciemment appliqués par l'enfant aux énoncés qui « entourent " la présentation initiale du mot, et aux termes dérivés à partir de ce mot.

Le peu que j'ai pu résumer ici de cette complexe procédure déductive et spontanée suffit déjà à nous montrer que ces principes et ces minithéorèmes ne peuvent pas être eux-mêmes « appris " (Piattelli-Palmarini, 1989). Ils ne peuvent pas l'être, parce qu'on ne voit pas quel genre de données en fournirait à l'enfant des indices indirects univoques, et parce que personne ne saurait les "enseigner » explicitement à l'enfant. Ils restent encore largement inconnus aux linguistes de profession. Là où ils sont un peu venus à la surface, ils défient toute traduction en des termes que l'enfant pourrait comprendre dans un langage qui lui soit accessible ${ }^{16}$. Le procès semble être entièrement guidé par des ressources internes,

15. Par exemple, "en une heure " signale que nous sommes en présence d'une seule action, tandis que " pendant une heure " (for an hour) si c'est une action (et non un état) signale que nous sommes en présence d'une série de répétitions d'une même action.

16. Cf. note 13. Il est impensable que l'on puisse enseigner à l'enfant (et même à une majorité d'adultes) à maitriser consciemment ce genre de critères. 
probablement innées, inaccessibles à la conscience, et en grande partie communes à toutes les langues naturelles. Les spécificités syntaxiques des différentes langues (j'en ai fourni un petit exemple ci-haut) deviennent accessibles en vertu de principes morpho-syntactico-sémantiques universaux convenablement " paramétrisés » (Chomsky, 1981, 1986; Roeper \& Williams, 1987). Dire que ces principes et ces contraintes pourraient provenir d'un enseignement implicite, indirect ou inconscient, reviendrait à poser un problème, et non pas à fournir une solution. En réalité, faire appel à un procès d'acquisition par " enseignement tacite ", ou implicite ou indirect, équivaut à re-présenter le même problème sous une forme superficiellement diffërente.

Venons-en maintenant à la phase terminale du processus d'acquisition lexicale.

\section{6. - L'ACQUISITION DE LA SIGNIFICATION « EXACTE »}

Les contraintes que je viens d'esquisser font un bon travail, un travail indispensable, mais elles ne suffisent pas à déterminer de façon univoque ce que le verbe 'ploncher' veut dire exactement. Au terme de toute cette analyse tacite, l'enfant peut bien rester sur sa faim sémantique, ne sachant toujours pas si l'aspect crucial de cette action de 'ploncher' est à chercher dans une certaine action (écraser, farcir, saler, suspendre, fumer), ou dans un ensemble ordonné ou non d'actions. Une composante cruciale manque encore ${ }^{17}$. L'enfant n'est pas encore "connecté avec le monde " de façon sémantiquement correcte en ce qui concerne ce terme. Le manque de connection causale est, nous le voyons, atomique et non pas général.

La composante sémantique qui permet de sélectionner le candidat spécifique, la signification « exacte ", est foncièrement constituée par une certaine suite d'actions et d'événements, sous une certaine description. Voici que les contraintes morpho-syntaxiques trouvent leur premier rôle causal. L'enfant est « connecté avec » un objet, une action, ou un événement, par une chaîne causale sémantique adéquate si, et seulement si, cet objet, cette action, cet événement, se présentent dans un certain encadre-

17. Lila R. Gleitman calcule que l'information morpho-syntaxique (ce qu'elle appelle le syntactic bootstrapping) fournit à elle seule une partie calculable de la signification d'un verbe (communication personnelle, voir aussi Lederer, Glemman \& GlemMAN, 1989). 
ment préalable (grosso modo, par un encadrement du type de celui que nous venons de voir). Les données perceptuelles doivent toujours être autant de faits-sous-description. "Regarder " simplement ce qui se passe ne suffit pas. Il faut se " connecter" avec ce qu'on observe au moyen d'une grille de repères linguistiques. La causalité sémantique est, comme il se doit, une causalité de dicto.

Aux données perceptuelles et syntaxiques non sollicitées, s'ajouteront les réponses aux questions pertinentes de l'enfant, souvent basées sur des raisonnements par contrefactuels («Maman, est-il possible de ploncher un poisson sur la plage?", " Peut-on ploncher aussi de la viande?", "Faut-il toujours avoir de la farine?»). Comme il se doit, la causalité sémantique doit pouvoir soutenir des raisonnements hypothétiques bien choisis. Tel est le deuxième rôle causal des données syntaxiques : jouer finement avec des possibilités et des situations imaginaires.

$\mathrm{Au}$ terme du processus, la signification exacte du nouveau mot est fixée dans le lexique de l'enfant. Un nouveau concept a été acquis. Ce qui confère au processus son caractère « atomique », c'est la nature extraordinairement spécifique de chacun de ces liens. Comme nous le verrons, il s'agit d'atomes qui ont une structure interne, mais qui "correspondent " un par un à des significations exactes. L'aspect atomique du processus, que je tiens à souligner, n'exclut pas que l'on doive se servir, pour identifier un de ces atomes sémantiques, d'autres significations, d'autres mots et d'autres concepts. Pour décider de quel lien il s'agit, dans chaque cas spécifique, il faut examiner aussi d'autres liens et utiliser des comparaisons fines, en ayant recours à toutes les ressources de la morphologie et de la syntaxe. Mais faire appel à ce qui «entoure " le concept n'implique, ni ne présuppose que le lien une fois établi soit causalement dépendant des autres liens examinés. Des données holistiques peuvent bien servir à établir et à corroborer une hypothèse parfaitement atomique. L'important est que l'identité et la spécificité du lien ne dépendent pas causalement du "tout». Elles peuvent en dépendre, toutefois, épistémiquement, de façon transitoire, pendant la phase d'acquisition.

\section{7. - L'ORGANISATION INTERNE DES ÉLÉMENTS LEXICAUX}

Les significations lexicales exactes ont deux composantes distinctes : une composante centrale et des informations associées, secondaires. Par exemple, il est essentiel à la signification de 'mariner' qu'il n'y ait pas de 
cuisson, tout comme il est essentiel à la signification de 'persuader' qu'il n'y ait pas d'obligation ${ }^{18}$. S'il est indispensable, pour soutenir ceci, que l'on réintroduise la distinction entre analytique et synthétique, je veux bien être parmi ceux qui le font (Chomsky, 1986). Il s'agit, toutefois, d'une distinction un peu différente. Comme le mot l'indique, quelque chose est analytique (dans l'acception habituelle du terme), si on y a en principe accès par la réflexion, tandis que beaucoup de ces composantes sémantiques centrales ne le sont pas ${ }^{19}$. Dans mon schéma atomique, il y a une structure interne de chaque atome, des vérités ponctuelles qui ne peuvent pas être niées, sauf à changer la signification de ce mot (sans changer le concept). Les plus importantes de ces vérités, toutefois, ne sont que rarement accessibles à l'entendement par simple auto-clarification, elles doivent être découvertes par une analyse linguistique comparée, par des données expérimentales relevant de la psychologie du développement, de la psycho-linguistique, des neurosciences cognitives (par exemple, avec l'étude de cas pathologiques), etc. Les différences d'âge, de langue maternelle, d'éducation générale peuvent fournir des données sémantiques très importantes. La composante centrale de la signification de chaque mot est de nature proprement sémantique. Étant largement inaccessible à l'analyse par autoclarification, elle ne coïncide donc pas avec la composante analytique. Elle est aussi sous-déterminée par toutes les connexions causales objectives que le sujet peut observer dans le monde. La signification ne doit pas se surcharger de connexions causales objectives $^{20}$. La composante centrale, en somme, n'est pas forcément bâtie

18. HALE \& KEYSER (1988) et PUSTEjovsky (1988) soutiennent que la structure événementielle fine des significations conceptuelles lexicales est causalement responsable des " projections " syntaxiques fondamentales d'un verbe (nombre de rôles thématiques, caractère de ces rôles, possibilité de « décharger " ces rôles d'une certaine manière, etc.). HigGinbothaM (1986) pense que seulement les rôles thématiques (ou " theta roles") sont syntaxiquement importants, tandis que la structure fine des événements reste foncièrement " invisible » à la syntaxe. Tous ces auteurs, il me semble, se trouvent d'accord sur l'extrême spécificité et la grande richesse des structures lexicales internes.

19. Supposons, par exemple, que dans la signification du verbe 'voler' it y ait une composante inéliminable de volonté soutenue (volonté du sujet, ou de celui qui a projeté un engin, ou de celui qui le pilote). Une mouche, un hélicoptère et un avion en papier 'volent', mais pas un boulet de canon, ni une flèche, ni une feuille morte. Supposons que ceci soit vrai. Il me semble que cette composante sémantique centrale du verbe 'voler' n'est pas nécessairement accessible à l'introspection, ni dictée par un critère d'ordre logique. Aucune règle de l'entendement ne nous interdirait de donner au verbe 'voler' la signification 'se mouvoir dans l'air', sans autre précision, de telle manière que les pierres qui tombent, un boomerang et la fumée 'volent' aussi (un grand nombre de cas semblables ont été proposés par CARTER).

20. Pour aller à la chasse au renard il faut avoir des bonnes raisons de supposer qu'il y ait des renards dans la région, mais la signification du verbe 'chasser' ne se décompose pas en termes d'anticipations, de suppositions et de probabilité. Par contre, on ne peut pas dire que l'on 'chasse ' quelque chose si on n'a pas l'intention de le capturer. Ceci est une composante cruciale de la signification du verbe 'chasser'. 
seulement sur des "vérités de raison " et sur des "vérités de fait». La composante analytique de la composante cruciale relève des principes de notre entendement, mais il y a aussi d'autres composantes, qui sont tacitement dictées par certaines structures modulaires contingentes de notre système cognitif (par exemple nos classes spontanées de similarité, nos prototypes naïfs et notre ontologie naïve). Elles ne demandent pas non plus à être vérifiées empiriquement par l'observation, car ce sont elles qui rendent l'observation possible en premier lieu. Leur fondement n'est pas dans les lois de la raison, comme c'est le cas des composantes strictement analytiques, mais dans notre nature humaine contingente.

Les vérités sémantiques centrales forment donc une classe particulière : leur composante analytique explique l'aspect déductif et les règles d'inférence logique mobilisées par le processus d'acquisition sémantique, tandis que la composante synthétique (largement tacite et ancrée sur des structures propres à notre espèce) explique l'aspect inductif, la recherche de confirmations et de réfutations spécifiques guidées par des hypothèses sémantiques spécifiques. La différence entre vérité analytique et vérité synthétique, que je tiens aussi à maintenir, ne coïncide donc pas avec la différence entre vérité sémantique et vérité d'ordre cognitif générique ou encyclopédique.

\section{8. - SÉMANTIQue LeXICALE ET CONNAISSANCE ENCYCLOPÉDIQUE}

Je tiens à souligner trois points :

1) En temps réel et d'un point de vue subjectif, toutes ces composantes (morphologique, syntaxique, sémantique, cognitive, spontanée, inductive, encyclopédique) sont inextricablement entremêlées et leurs contributions respectives sont difficilement accessibles à l'introspection. Néanmoins, elles doivent en principe, et elles peuvent de facto, demeurer séparées dans une analyse scientifique. Il est clair, par exemple, que certaines composantes sont universelles, que d'autres sont spécifiques à une langue donnée, d'autres encore propres à une culture, ou à un certain groupe particulier, ou même à un individu donné. L'analyse scientifique parvient déjà à tracer des différences entre toutes ces contributions et à voir (ou entrevoir) les contraintes qui agissent sur chacune d'elles.

2) Les composantes morphologique, syntaxique et sémantique sont contraintes aussi bien par des principes de la grammaire universelle, commune à toutes les langues, que par des règles locales, propres à chaque langue (comme c'est le cas pour la middle construction en 
anglais); la théorie linguistique actuelle postule un processus de « fixation de paramètres " pour rendre compte de cette relation entre universel et particulier.

3) La composante sémantique est enracinée dans le savoir encyclopédique de chaque sujet, mais elle est en même temps plus vaste et plus restreinte que ce que chacun "sait " du monde.

Sur ce dernier point, j'ajoute que le savoir sémantique individuel excède le savoir encyclopédique individuel, parce que chacun de nous est " fondé de pouvoir » en ce qui concerne l'usage propre, plein et adéquat de beaucoup de termes de notre langue (et même de beaucoup de termes de notre idiolecte privé), en dépit d'un accès seulement partiel à la signification exacte et à l'usage collectif attitré (par exemple, chez les scientifiques professionnels ou les experts), et en dépit de connaissances encyclopédiques souvent presque négligeables sur ces mots (Higginbotham, 1988; Piattelli-Palmarini, 1988a). Sous un angle fort different de celui de Higginbotham et du mien, cette " division du travail » entre les experts et les locuteurs ordinaires fonde aussi la théorie causale de la référence de Saul Kripke (1972) et de Hilary Putnam (1975). Mais le savoir sémantique est aussi plus restreint, car seulement un sousensemble spécifique et limité de tout ce que nous savons sur les chats ou sur les objets volants est mobilisé dans la signification des mots 'chat' ou 'voler' ${ }^{21}$. En outre, il est crucial de souligner une fois de plus que le savoir sémantique exploite des éléments de connaissance tacite inaccessibles à l'introspection. Par exemple, comme nous l'avons vu ci-dessus, il est parfois sémantiquement capital que l'action exprimée par un verbe soit mesurable en ce qui concerne son " argument interne ", mais pas son "argument externe", ou que la réfërence à un événement qui " culmine " soit obligatoire, ou que l'objet de l'action soit "affecté " d'une certaine façon, et ainsi de suite. Il s'agit de notions techniques, propres à la linguistique actuelle, qui échappent à la simple autoclarification introspective. Nous sommes censés avoir un accès introspectif à nos connaissances encyclopédiques, mais pas à ce genre de connaissances sémantiques. En effet, les connaissances sémantiques sont acquises, utilisées et mises à l'épreuve tacitement, sans trop de contrôle volontaire. Avec la plus grande facilité, un formidable appareil individuel

21. Par exemple, je considère qu'il suffit à un locuteur, pour pouvoir utiliser correctement dans son discours le terme «fractal ", qu'il sache (en gros) qu'il s'agit d'entitès mathématiques « découvertes » récemment, qui trouvent de plus en plus d'applications pratiques et que leur représentation graphique rappelle vaguement les vitraux d'une cathédrale. Par contre, un deuxième locuteur qui croit que les fractales sont une sorte de particules élémentaires, ne sait pas de quoi il parle, même s'il possède des renseignements encyclopédiques plus détaillés (et fondamentalement vrais) sur Benoît Mandelbrot, sur la date de leur " découverte », etc. 
et de source interne sait comment se servir de données multiples (perceptuelles, linguistiques, déductives, inductives) pour parvenir à ses fins.

Il est utile de recourir, en sémantique, à maintes notions traditionnelles (par exemple à la distinction classique entre analytique et synthétique, à la notion de critère nécessaire et/ou suffisant, à la notion de fixation d'hypothèses, à la notion de définition, et ainsi de suite). Toutes sont utilisables en sémantique, mais aucune ne peut rendre compte, par ses seuls moyens, de la nature de la signification. Une meilleure compréhension des rapports entre savoir sémantique et savoir encyclopédique mobilise tous ces concepts, mais oblige aussi à cerner (rien de moins que) l'articulation entre les systèmes morpho-syntaxiques, perceptuels, cognitifs au sens large, et les systèmes de croyances, aussi bien collectives qu'individuelles. Les multiples tentatives pour réduire la sémantique à un et un seul de ces systèmes ont échoué. Ce qui n'a rien d'étonnant, vu la nature du problème.

Avant de réfléchir sur les conséquences philosophiques de ce schéma naturaliste, individualiste, internaliste et atomique, il reste à souligner que la signification " exacte » sera aussi exacte qu'il le faut, pas moins, et pas plus. Ceci est un point capital. Ma représentation mentale du mot ' mariner' n'est pas exactement la même que celle de Pierre. Néanmoins, nous entendons la même chose par ce mot. Nous avons la même représentation sémantique du mot 'mariner', en dépit de nombreuses différences entre nos images mentales individuelles (non seulement « en général ", mais en ce qui concerne l'action spécifique de mariner). Aussi fine soit-elle, la connexion sémantique avec le monde n'impose ni ne présuppose l'identité psychologique des contenus mentaux individuels atomiques. Je laisse ici au niveau d'un simple énoncé ce qui pourrait être montré en détail : c'est-à-dire, que la causalité sémantique, selon mon schéma, est précisément celle qui permet juste le degré de variabilité qu'il nous faut parmi les représentations mentales individuelles soutenues (ou subsumées) par une même signification sémantique exacte. Les limites de cette variabilité coïncident avec le flou des informations collatérales que chacun possède sur un certain objet, ou sur une certaine action ${ }^{22}$.

Ceci s'oppose, il est clair, à l'approche holiste de la sémantique, selon

22. Si Pierre croyait la cuisson indispensable pour 'mariner', ce mot aurait, dans l'idiolecte de Pierre, une autre signification que dans mon idiolecte. Si, par contre, Pierre croyait que pour 'mariner' il est indispensable de tremper quelque chose dans le vinaigre (pas dans du citron, ou de la sauce, etc.), il y aurait, entre Pierre et moi, seulement une différence d'ordre encyclopédique. Nous aurions la même sémantique pour le mot 'mariner' (nous en aurions le même concept), en dépit de la différence de nos croyances sur le processus (pour une analyse exhaustive de ces cas, sous une perspective différente de la mienne, voir les travaux de Burge). 
laquelle toutes les informations collatérales (toutes nos connaissances encyclopédiques) sont en principe inséparables de la signification proprement dite. Les holistes soutiennent qu'il n'y a aucune distinction de principe entre la signification (meaning) d'un mot et les croyances (beliefs) portant sur l'état de choses que ce mot symbolise. Selon eux, une signification "n'est rien d'autre que " le faisceau variable des croyances que chaque locuteur entretient et qui sont vaguement véhiculées par ce mot. Il est typique de cette approche de nier, en principe également, toute séparation entre syntaxe, sémantique et pragmatique et de nier avec force la séparation entre synthétique et analytique ${ }^{23}$. Pour un vrai holiste, il n'y a jamais la signification " exacte " d'un terme isolé. Il n'y a que des réseaux de significations se soutenant les unes les autres et s'interdéfinissant par coopération.

Cette extrême libéralité sémantique n'a pas satisfait tout le monde et on a cherché à y mettre quelques limites. Le projet devint alors non pas de réintroduire une distinction entre le synthétique et l'analytique, mais de régimenter l'usage des concepts et des mots par des critères robustes de cohérence inférentielle subjective (Tyler Burge), ou par des chaines causales objectives, largement indépendantes des contenus mentaux du locuteur particulier (Putnam et Kripke). Il faut admettre que la démarcation entre composantes cruciales et composantes secondaires de la signification a connu des difficultés considérables. Je ne peux pas les passer sous silence.

\section{9. - OBJECTIONS ET CONTRE-OBJECTIONS}

L'espoir de dégager un noyau central fixe dans la signification d'un mot a été fortement entamé par la démonstration de Putnam selon laquelle il n'est même pas indispensable à la signification du mot 'chat' de croire qu'il s'agit d'un animal (et pas, par exemple, d'un robot), et par celle de Fodor selon laquelle 'tuer' ne peut pas être dérivé sémantiquement de 'causer la mort'. En sémantique, Putnam et Kripke ont montré l'insuffisance foncière des critères nécessaires et suffisants (Kripke, 1972; Putnam, 1970, 1975), tandis que Fodor a montré l'insuffisance foncière des définitions (Fodor, 1970, 1987). Ces auteurs en ont tiré des conclusions opposées: pour Putnam et Kripke, les significations forment un

23. Quant à la morphologie et à ses effets sémantiques indéniables, il me semble que les holistes n'y aient même jamais pensé. 
réseau en transformation continue, engendré par une interaction causale collective et objective avec le monde; pour Fodor, les significations sont atomiques, engendrées (une par une) par une interaction causale, bien déterminée, individuelle et strictement atomiste. A la différence de ce que je soutiens ici, Fodor pense qu'il n'y a pas de structure lexicale interne, et que chaque mot, chaque concept, est comme une ampoule indécomposable, séparée des autres ampoules. Elle s'allume dans notre tête si, et seulement si, nous sommes connectés causalement de la bonne manière avec une certaine région (ou un certain aspect) spécifique du monde ${ }^{24}$. La sémantique est, pour Fodor, une propriété intrinsèque du «mentalais " (the language of thought) qui se reflète seulement de manière indirecte dans les traductions standard dans les différentes langues naturelles et constitue les contraintes objectives sur la syntaxe de ces traductions. Ce que j'ai appelé ici sémantique serait, pour Fodor, de la pure syntaxe des langues naturelles, sous un autre nom.

Certaines de ces objections me semblent insoutenables, certaines autres justes, mais contournables. L'hypothèse d'une construction foncièrement collective et holistique de la sémantique me semble réfutée par la découverte d'un nombre croissant de ressemblances (et identités) morpho-syntactico-sémantiques fondamentales entre toutes les langues, y compris les langues historiquement et géographiquement éloignées. Il faut en conclure que la prétendue chaîne causale collective est inexistante, ou interrompue depuis fort longtemps, et qu'on ne peut pas expliquer ces ressemblances profondes, entre des langues objectivement isolées l'une de l'autre, par une transmission collective de bouche à oreille. Qui plus est, comme je l'ai indiqué, la nature même des principes morphosyntactico-sémantiques fondamentaux est telle que leur accessibilité à la conscience est pratiquement nulle. La transmission ne peut pas se fonder sur un enseignement explicite. Invoquer la possibilité d'un enseignement implicite (même là où elle est concevable) n'est qu'une autre façon de poser le problème. L'efficacité et la vitesse du processus d'acquisition lexicale peuvent s'expliquer seulement par une utilisation massive par

24. Il n'y a pas d'électrons et de protons dans les atomes sémantiques de Fodor. La connexion causale qui fonde la sémantique à la Fodor n'est pas quelque chose à laquelle on doit chercher un « accès » psychologique, ou linguistique, quelconque. C'est simplement une relation objective qui nous implique, quelque chose qui nous arrive, et non pas une relation que nous engendrons par nos opérations mentales. Celles-ci se mobilisent par un tout autre mécanisme, celui qui engendre nos attitudes mentales (croire, redouter, espérer, nier, affirmer, etc.) vis-à-vis de nos concepts. Tout ce que j'ai dit plus haut sur le lexique et son acquisition est, pour Fodor, un aspect de la traduction de nos concepts du 'mentalais' dans une langue naturelle (anglais, chinois, etc.). Comme il l'admet lui-même, certains aspects de sa conception actuelle du lien causal objectif entre le monde et les atomes sémantiques du mentalais sont (curieusement) assez proches d'une conception skinnerienne (FODOR, 1989). 
chaque locuteur de contraintes non apprises, indépendantes du contexte et de nature strictement tacite.

A la différence de Fodor, je suis toutefois persuadé que les atomes sémantiques ont une structure interne et que certaines composantes sont cruciales pour la signification. Si un locuteur n'a pas compris qu'elles sont cruciales, il n'a pas vraiment acquis la signification. Par contre, d'autres composantes sont accessoires et pour elles j'admets une large variabilité de croyances et d'usages entre des locuteurs qui attachent, néanmoins, la même signification aux mêmes mots.

Un problème de redondance se manifeste d'emblée, puisque la frontière entre ce qui est crucial et ce qui est accessoire dépend du terme en question. A la limite, il se peut bien qu'il y ait autant de composantes cruciales qu'il y a de concepts mono-lexicalisables. Je considère toutefois le problème de la redondance comme un pseudo-problème, car ici, comme partout ailleurs en biologie, il ne faut pas présupposer de parcimonie (Piattelli-Palmarini, 1985), ni chercher à tout prix une économie de moyens. Le rôle causal des composantes sémantiques cruciales n'est pas celui de minimiser, ou de réduire, la tâche d'acquisition, le poids de mémorisation, ou la complexité d'usage. Leur rôle est de fonder l'invariance de la signification et la spécificité de la causalité sémantique, en dépit des différences cognitives individuelles.

Pourtant Putnam a raison: les composantes cruciales ne sont pas identiques aux critères nécessaires et suffisants de nature encyclopédique, structurale ou micro-physique. Fort peu de locuteurs savent ce qui est biologiquement nécessaire et suffisant pour décider qu'un animal est un chat, physiquement nécessaire et suffisant pour décider que quelque chose est en aluminium, pragmatiquement nécessaire et suffisant pour décider quand il y a eu une 'interférence'. Tous les locuteurs, pourtant, savent ce que signifient 'chat', 'aluminium', 'interférence'. Ce qui semble crucial, pour saisir la signification de ces termes, c'est plutôt (en première approximation) la propriété de miauler et d'avoir des moustaches, la brillance et la légèreté du matériel, l'entrave à une action entamée et conduite par autrui. Je ne me préoccupe pas de mieux "définir» ces composantes, car il ne s'agit pas non plus, comme Fodor l'a justement souligné, de définitions. Une définition utilise une périphrase bien polie, constituée par des mots qui nous sont tous déjà explicitement connus. Les composantes sémantiques cruciales, par contre, peuvent nous être connues seulement de façon tacite, sans qu'elles soient "effables" (tandis qu'une définition doit l'être) et sans qu'elles soient accessibles à l'introspection (tandis qu'un critère nécessaire et/ou suffisant doit l'être). Ces composantes font l'objet d'une veritable recherche scientifique empirique, guidée par des théories morphologiques et syntaxiques profondes 
et détaillées et, le plus souvent, par une comparaison avec des langues fort « exotiques". Ces composantes cruciales se révèlent parfois fort différentes de ce que notre intuition nous suggère ${ }^{25}$. La sémantique des langues naturelles mobilise tacitement aussi toutes sortes de "théories" naìves sur le monde, qui sont bien les nôtres, mais sur lesquelles nous exerçons peu de contrôle conscient (Carey, 1985 ; Jackendoff, 1983 ; Keil, 1979 ; Macnamara, 1982 ; Markman, 1989; McCloskey, 1983 ; Nersessian \& Resnick, 1989 ; Spelke, 1988).

\section{0. - LES COMPOSANTES CRUCIALES DES ATOMES SÉMANTIQUES}

L'acquisition portera, non pas pour des raisons logiques ou pour des nécessités d'ordre pragmatique, mais pour des raisons contingentes à notre espèce, d'abord sur les ensembles naturels typiques (natural kinds) et sur leurs attributs fondamentaux, sur les actions fondamentales et sur les types d'événements fondamentaux. Ces catégories ontologiques spontanées correspondent, dans l'ordre, aux catégories syntaxiques et morphologiques de syntagme nominal ('ta sceur', 'les bébés', 'un ours', etc.), de syntagme verbal ('s'en va', 'est arrivé', 'disons', etc.) et syntagme adverbial-modal ('lentement', 'hier', 'sans bruit', etc.). L'ontologie spontanée pré-sélectionne les unités saillantes et leurs attributs (manifestes ou cachés) ${ }^{26}$. La psychologie spontanée sélectionne les actions saillantes et permet d'en deviner les causes internes, volontaires ou involontaires. L'usage de plus en plus subtil des quantificateurs syntaxiques ('le', 'un', 'plusieurs', etc.), des quantificateurs logiques ('tous', 'aucun', 'un seul', 'quelques', etc.) et des quantificateurs adver-

25. Par exemple, en dépit de ce que pensent beaucoup de locuteurs de l'anglais, ce qui est crucial à la signification du terme crunchy n'est point une certaine consistance matérielle, mais un certain son crépitant que l'on doit entendre dans la bouche. Les locuteurs du français semblent être mieux situés pour capturer intuitivement cette composante sémantique du mot correspondant 'croquant'. Pourtant, les deux termes sont la traduction fidèle l'un de l'autre. Ces diffèrences entre les langues sont souvent profondes et très révélatrices, mais il serait insensé d'en tirer des arguments pour une quelconque intraduisibilité systématique entre les langues naturelles. Une meilleure compréhension de la sémantique permet, entre autre, d'expliquer les raisons du phénomène opposé : il y a traduisibilité systématique entre n'importe quelle langue naturelle et $n$ 'importe quelle autre langue naturelle. Les cas d'authentique intraduisibilité sont exceptionnels, quoique fort intéressants pour le thécricien.

26. Déjà à l'âge de deux ans et demi un enfant sait parfaitement quand un terme fait référence à un objet (parapluie, marteau) et quand un terme fait référence à un agrégat, ou une substance, (pommade, sable, etc.) (travaux de Susan Carey et Nancy Soja). 
biaux ('souvent', 'jamais', 'avant de', 'pendant que', etc.) permet à l'enfant de saisir, et de proposer à ceux qui l'écoutent, des situations réelles et hypothétiques de plus en plus fines. D'autres termes et d'autres significations deviennent accessibles (conditionnels, conjonctions, disjonctions, relativisations, etc.). Le bootstrapping syntaxique permet d'aiguiser les nouvelles acquisitions lexicales jusqu'à un degré de raffinement comparable à celui de l'adulte. L'enfant sait tacitement, sans que personne ne le lui ait jamais "enseigné ", que seules certaines connexions sont cruciales et qu'il lui faut, cas par cas, les détecter. Les ressources internes, aussi bien cognitives que linguistiques, sélectionnent ce qui peut constituer une composante cruciale de cette signification lexicale ${ }^{27}$. Comme le souligne Higginbotham (Higginbotham, 1988), il peut y avoir un élément indiciel (indexical) irréductible dans le mécanisme causal sémantique. On pointe vers un terme ou une expression de la langue $e t$ vers quelque chose de spécifique dans le monde, et on laisse les ressources internes compléter la définition de ce vers quoi l'on pointe, le degré de détails, la finalité de l'opération, etc. Le questionnement pertinent est une méthode fondamentale pour affiner le lien causal entre le sujet et ce qui vient d'être montré. On sait bien qu'il n'y a point d'ostension sans conceptualisation et les ressources internes fournissent juste la conceptualisation nécessaire ${ }^{28}$, pour que l'acte d'ostension soit un acte sémantiquement réussi. Ni plus, ni moins.

L'idée centrale de mon schéma est qu'une fois détectées, les composantes cruciales d'un concept vont constituer le lien causal cherché entre la signification et cet 'état-de-choses-sous-description'. Le lien est ponctuel, spécifique et capable d'engendrer par déduction tacite toutes sortes de conséquences spécifiques, à plusieurs niveaux (morphologique, syntaxique, sémantique et cognitif). La composante syntaxique se charge, bien sûr, d'agencer les mots en énoncés de plus en plus longs et complexes, doués de significations de plus en plus subtiles. On parvient à décrire des états de choses qui sont vrais ou faux, ou probablement

27. L'intention du constructeur et l'usage habituel prévu seront des bons candidats pour un terme qui fait référence à un produit industriel ou artisanal, mais pas pour le nom d'un animal, tandis que le changement effectué dans l'état d'intégrité matérielle d'un objet sera un bon candidat pour un verbe transitif de manipulation, mais pas pour un verbe intransitif faisant référence au temps atmosphérique. Et ainsi de suite.

28. C'est par des ressources internes que chacun sait que l'adjectif 'blanc', appliqué à un œuf ou à un mur, n'implique pas qu'ils soient blancs de part en part, tandis que l'adjectif 'jaune' appliqué à l'or implique que cette substance soit jaune de part en part. Il serait impossible de connecter causalement l'enfant au monde en lui " montrant " quoi que ce soit, s'il était dénué de ces hypothèses restrictives spontanées. De toute évidence, ces choses-là personne ne nous les a "enseignées", car les enseigner présupposerait toujours que des connaissances tout aussi complexes que celle-ci soient déjà accessibles. 
vrais, ou probablement faux, à partir d'atomes qui sont « vrais de " (true of) certains objets ou de certains événements atomiques.

Le locuteur, en somme, possède tous les outils qui lui sont nécessaires et suffisants pour " connecter» un certain énoncé avec un certain état de choses. En vertu de sa nature humaine et de la spécificité linguistique de son environnement (qu'il doit " apprendre » en même temps qu'il enrichit son lexique), il se trouve causalement connecté avec le monde, et avec ses interlocuteurs, par des mécanismes efficaces, subtils et hautement sélectifs. La causalité dont je parlais au départ, celle qui explique nos actions spécifiques par des expressions spécifiques de nos langues naturelles, est enracinée dans les mécanismes que je viens d'esquisser. Elle est naturelle, car elle est ancrée dans notre patrimoine biologique, mais elle n'est pas réductible à la physique, ni à aucune version naïve de la biologie évolutionniste. Comme nous venons de le voir, elle est compatible avec les mécanismes détaillés par les sciences physiques et biologiques, mais elle fait l'objet d'une autre science naturelle spécialisée. A mon avis, se méfier d'un pareil projet d'une sémantique naturelle à cause de l'impossibilité de sa réduction aux lois de la physique serait comme se méfier des règles de priorité dans l'art de naviguer à cause de l'impossibilité de trouver une traduction moléculaire de termes comme « babord amûres ".

\section{CONCLUSION}

Ainsi les atomes sémantiques possèdent une structure interne spécifique, avec des composantes cruciales, invariables, et des composantes secondaires, variables d'un locuteur à l'autre. Ne pas saisir cette différence entre les composantes, ou confondre les unes avec les autres, signifie ne pas « posséder " une certaine signification, ne pas posséder un certain concept, ne pas être connecté avec le monde de façon sémantiquement correcte en ce qui concerne ce concept, ce terme lexical. Notre capacité à saisir ces composantes constitue la base naturelle de la causalité sémantique. Ce processus de sélection sémantique, il faut le penser répété de façon spécifique pour chacun de nos concepts fondamentaux, c'est-à-dire, pour chacun des termes de notre lexique. Il s'agit, au plus, d'un répertoire de quelques centaines de milliers de termes. Un répertoire qui est ouvert à de nouvelles insertions, pourvu que de fortes contraintes structurales soient respectées. Chaque terme possède sa spécificité et les différentes langues ont des moyens un peu différents de 
sélectionner les composantes cruciales et les composantes secondaires, en créant des répertoires lexicaux légèrement différents. Ces différences sont toujours limitées par des contraintes universelles, ce qui veut dire que seul un sous-ensemble de tout ce qui nous est concevable est aussi monolexicalisable.

Jusqu'à ces dernières années, les différences lexicales entre les langues (les mots "intraduisibles») ont retenu l'attention des linguistes beaucoup plus que les profondes et remarquables similitudes entre tous les lexiques de toutes les langues naturelles. Cette similitude, étudiée à un niveau de profondeur morpho-syntaxique et sémantique adéquat, offre déjà quelques généralisations scientifiques intéressantes.

Ces généralisations ne sont que partielles et encore provisoires, mais elles démontrent déjà que nous sommes naturellement équipés pour saisir ces nombreuses structures spécifiques, sur la base de données limitées, épisodiques et apparemment largement insuffisantes. L'ensemble de nos systèmes langagiers et beaucoup de nos théories spontanées du monde sont mobilisés par le processus d'acquisition. Ces différents systèmes linguistiques et cognitifs, en grande partie modulaires, restent distincts dans leurs principes fonctionnels et architecturaux, mais ils interagissent étroitement et finement dans l'acquisition du lexique.

Qu'il y ait autant de structures spécifiques fines qu'il y a de significations monolexicales (ou concepts «primitifs") possibles ne devrait pas nous étonner. Un ordre de grandeur de quelques centaines de milliers, ou quelques millions, de structures spécifiques pour un répertoire biologique est parfaitement normal (Piattelli-Palmarini, 1986, 1989). Je me permets de souligner que chacun de nos organismes possède à la naissance un bien plus grand nombre de types d'anticorps. Il s'agit de structures de haute complexité, qui sont tout aussi spécifiques, tout aussi diversifiées, et tout aussi innées, que nos concepts dits primitifs.

Massimo Ptattelli-Palmarini **,
Center for Cognitive Science, M.I.T.,
Cambridge, Massachusetts.

** Je remercie François Dell, Chantal Hunt et Jean-Michel Roy pour leur lecture critique d'une première version de ce manuscrit. 


\section{BIBLIOGRAPHIE}

BAKER (Mark), 1988, Incorporation : A Theory of Grammatical Function Changing, Chicago, III., Chicago University Press.

BOLtzMANN (Ludwig), 1904, "On a Thesis of Schopenhauer", in B. McGuinNEsS, ed., 1974, Theoretical Physics and Philosophical Problems, Dordrecht, Holland, D. Reidel.

Campbell (Donald T.), 1974, " Evolutionary Epistemology ", in P.A. SCHILPP, ed., The Philosophy of Karl Popper, La Salle, I11., Open Court, p. 413-463.

CAREY (Susan), 1985, Conceptual Change in Childhood, Cambridge, MA, Bradford Books/MIT Press.

CARTER (Richard J.), 1980, "La notion d'explication en sémantique ", Langue française, 46.

CARTER (Richard J.), $1984 \mathrm{a}$, "Compositionality and Polysemy ", in LEVIN \& TENNY, eds, 1988, p. 167-204.

CARTer (Richard J.), 1984b, “ On Movement ", in LeVIN \& TENNY, eds, 1988, p. 231-252.

CARTER (Richard J.), 1984 c, « Sous-catégorisation et régularités sélectionnelles », Communications, 40, p. 181-209.

CHOMSKY (Noam), 1955, "Transformational Analysis ", University of Pennsylvania, Thèse de doctorat, in CHOMSкY, 1985.

Chomsky (Noam), 1959, "A Review of B.F. Skinner's 'Verbal Behavior'", Language, 35/1, p. 26-58.

ChOMSKy (Noam), 1981, Lectures on Government and Binding : The Pisa Lectures, Dordrecht, Holland, Foris.

Chomsky (Noam), 1985, The Logical Structure of Linguistic Theory, Chicago, Ill, The University of Chicago Press.

CHOMSKY (Noam), 1986, Knowledge of Language : Its Nature, Origin, and Use, Convergence, New York, Praeger Scientific.

DENNETT (Daniel C.), 1983, «Intentional Systems in Cognitive Ethology: The 'Panglossian Paradigm' Defended", The Behavioral and Brain Sciences, 6/3, p. 343-390.

DenNETt (Daniel C.), 1987, The Intentional Stance, Cambridge, MA, Bradford Books/MIT Press.

DenNeTt (Daniel C.), 1988, "Ways of Establishing Harmony ", in B. MCLAughLIN, ed., Essays in the Honour of Fred Dretske (en préparation).

DowTY (David R.), 1979, Word Meaning and Montague Grammar, Dordrecht, Holland, D. Reidel.

Dretske (Fred), 1981, Knowledge and the Flow of Information, Cambridge, MA, Bradford Books/MIT Press. 
DreTsKe (Fred), 1986, « Misrepresentation », in R. BodGAN, ed., Belief, Oxford, Oxford University Press.

DRETSKE (Fred), 1988, Explaining Behaviour : Reasons in a World of Causes, Cambridge, MA, Bradford Books/MIT Press.

ELMAN (Jeffrey L.), 1989, "Representation and Structure in Connectionist Models", in Center for Research in Language Technical Report 8903, La Jolla, CA, University of California, San Diego.

Fillmore (Charles), 1968, "The Case for Case", in E. BACH \& R. HARMS, eds, Universals in Linguistic Theory, New York, Holt, Rinehart \& Winston, p. 188.

FodoR (Jerry A.), 1970, "Three Reasons for not Deriving 'Kill' from 'Cause to Die' ", Linguistic Inquiry, 1, p. 429-438.

Fodor (Jerry A.), 1987, Psychosemantics, Cambridge, MA, Bradford Books/MIT Press.

FoDor (Jerry A.), 1989, " A Theory of Content ", Department of Philosophy, City University of New York, manuscrit non publié.

Fodor (Jerry A.) \& Pylyshyn (Zenon), 1988, « Connectionism and Cognitive Architecture : A Critical Analysis ", in S. Pinker \& J. Mehler, eds, Connections and Symbols, Cambridge, MA, Bradford Books/MIT Press.

GlemtMaN (Lila R.), 1986, « Biological Dispositions to Learn Language ", in W. Demopoulos \& A. MARRAS, eds, Language Learning and Concept Acquisition : Foundational Issues, Norwood, NJ, Ablex.

Goldstone (Robert L.), Gentner (Derdre) \& MEdin (Douglas L.), 1989, « Relations Relating Relations", in 11th. Annual Conference of the Cognitive Science Society, Ann Arbor, Michigan, Lawrence Erlbaum Associates, vol. XI, p. 131-138.

Grimshaw (Jane), 1979, "Complement Selection and the Lexicon ", Linguistic Inquiry, 10/2.

GRIMSHAw (Jane), 1982, “ On the Lexical Representation of Romance Reflexive Clitics ", in J. BRESNAN, ed., The Mental Representation of Grammatical Relations, Cambridge, MA, MIT Press.

HALE (Kenneth) \& KeYSER (Samuel Jay), 1988, « Explaining and Constraining the English Middle ", in C. TENNY, ed., Studies in Generative Approaches to Aspect, Lexicon Project Working Papers, $n^{\circ} 24$, Cambridge, MA, MIT Center for Cognitive Science, p. 41-57.

Hale (Kenneth) \& KeYSER (Samuel Jay), 1989, «The Syntactic Character of Thematic Structure », manuscrit non publié, Lexicon Project, MIT Center for Cognitive Science.

Higginbotham (James T.), 1985, «On Semantics », Linguistic Inquiry, 16/4, p. 547-593.

HigGinBOTHAM (James T.), 1986, « Elucidations of Meaning ", Linguistics and Philosophy, 12/3.

HigginbothaM (James T.), 1988, " Knowledge of Reference ", in A. GEORGE, ed., Reflections on Chomsky, Oxford, Basil Blackwell.

JACkendoff (Ray), 1983, Semantics and Cognition, Cambridge, MA, MIT Press.

JACKENDOFF (Ray), 1987, "The Status of Thematic Relations in Linguistic Theory », Linguistic Inquiry, 18/3. 
KeIL (Frank C.), 1979, Semantic and Conceptual Development : An Ontological Perspective, Cambridge, MA, Harvard University Press.

KAYNE (Richard S.), 1975, French Syntax, Cambridge, MA, MIT Press.

KRIPKE (Saul), 1972, Meaning and Necessity, Oxford, Oxford University Press.

LANDAU (Barbara) \& GleITMAN (Lila R.), 1985, Language and Experience : Evidence from the Blind Child, Cambridge, MA, Harvard University Press.

LASNIK (Howard) \& URIagereka (Juan), 1988, A Course in GB Syntax : Lectures on Binding and Empty Categories, Cambridge, MA, MIT Press.

Lederer (Anne), Glettman (Henry) \& Glemman (Lila R.), 1989, « Syntactic Bootstrapping : Are the Data for a Deductive, Principle-Driven Verb Learning Procedure Available to Children?", communication présentée à The 14th. Annual Conference on Language Development, Boston University, oct. 1989.

Levin (Beth) \& Tenny (Carol), eds, 1988, On Linking : Papers by Richard Carter, Lexicon Project Working Papers, $\mathrm{n}^{\circ}$ 25, Cambridge, MA, MIT Center for Cognitive Science.

LoAR (Brian), 1981, Mind and Meaning, Cambridge, U.K., Cambridge University Press.

LORENZ (Konrad), 1941, « Kants Lehre vom Apriorischen im Lichte gegenwärtiger Biologie ", Blätter für deutsche Philosophie, 15, p. 94-125.

Lorenz (Konrad), 1982, "Kants Doctrine of the A Priori in the Light of Contemporary Biology », trad. de LORENZ, 1941, in H.C. PlotKIN, ed., Learning, Development and Culture : Essays in Evolutionary Epistemology, New York, John Wiley \& Sons.

MacNamara (John), 1982, Names for Things: A Study of Human Learning, Cambridge, MA, Bradford Books/MIT Press.

Markman (Ellen M.), 1989, Categorization and Naming in Children : Problems of Induction. Cambridge, MA, Bradford Books/MIT Press.

MARSLEN-Wilson (William) \& TYler (Lorraine), 1980, « The Temporal Structure of Spoken Language Understanding ", Cognition, 8, p. 1-71.

MAY (Robert), 1985, Logical Form : Its Structure and Derivation, Cambridge, MA, MIT Press.

MCCloskey (Michael), 1983, "Naive Theories of Motion », in D. GentNER \& A.L. SteVEns, eds, Mental Models, Hillsdale, NJ, Lawrence Erlbaum.

MILlER (George A.), 1986, " Dictionaries in the Mind ", Language and Cognitive Processes, 1/3, p. 171-185.

Miller (George A.) \& Gildea (Patricia M.), 1987, « How Children Learn Words ", Scientific American, 257/3, p. 94-99.

Millikan (Ruth Garrett), 1984, Langage, Thought, and Other Biological Categories, Cambridge, MA, Bradford Books/MIT Press.

Millikan (Ruth Garrett), 1986, « Thoughts without Laws : Cognitive Science with Content ", Philosophical Review, 95/1, p. 47-80.

Millikan (Ruth Garrett), 1989, "Truth Rules, Hoverflies, and the KripkeWittgenstein Paradox ", Philosophical Review, à paraître en 1990.

NerSESSIAN (Nancy J.) \& RESNICK (Lauren B.), 1989, « Comparing Historical and Intuitive Explanations of Motion: Does 'Naive Physics' Have a Struc- 
ture?", in 11th. Annual Conference of the Cognitive Science Society, Ann Arbor, Michigan, Lawrence Erlbaum Associates, p. 412-418.

PeIRCE (Charles Sanders), 1896, "The Scientific Attitude and Fallibilism ", in J. BuCHLer, ed., 1955, Philosophical Writings of Peirce, New York, Dover.

PESETSKY (David), 1985, " Morphology and Logical Form ", Linguistic Inquiry, 16/2, p. 193-246.

Pesetsky (David), 1987, « Binding Problems with Experiencer Verbs », Linguistic Inquiry, 18, p. 126-140.

Peтrтot (Jean), 1985, Morphogenèse du sens. I, Paris, Presses universitaires de France.

PETrTot (Jean), 1989, « Hypothèse localiste, modèles morphodynamiques et théories cognitives : remarques sur une note de 1975 ", à paraitre dans Semiotica.

Piatielli-Palmarini (Massimo), 1985, «The Waning of Parsimony », Scientia, numéro spécial "La vita e la sua storia", sous la dir. de L. BullinI, M. Ferraguti, A. Oliverio \& F. Mondella, p. 265-279.

Piattelli-Palmarini (Massimo), 1986, "The Rise of Selective Theories : A Case Study and some Lessons from Immunology ", in W. DEMOPOULos \& A. MARRAS, eds, Language Learming and Concept Acquisition : Foundational Issues, Norwood, NJ, Ablex.

Piattelli-Palmarini (Massimo), 1988a, "Can We Rationally Believe Something We Do Not Quite Understand?", in Actes du colloque Gli Stili dell' Argomentazione, Modena, Fondazione San Carlo, à paraître.

Piattelli-Palmarini (Massimo), 1988b, « Not on Darwin's Shoulders : A Critique of Evolutionary Epistemology ", in Boston Colloquia for the Philosophy of Science, Boston, MA, à paraître.

Piattelli-Palmarini (Massimo), 1989, "Evolution, Selection and Cognition : From 'Learning' to Parameter Setting in Biology and in the Study of Language ", Cognition, 31/1, p. 1-44.

PINKER (Steven), 1989, Learnability and Cognition : the Acquisition of Argument Structure, Cambridge, MA, MIT Press.

POPPER (Karl R.), 1972, Objective Knowledge : An Evolutionary Approach, Oxford, Clarendon Press.

Pustejovsky (James), 1988, "The Geometry of Events ", in C. TENNY, ed., Studies in Generative Approaches to Aspect, Cambridge, MA, MIT Center for Cognitive Science, p. 19-39.

Putnam (Hilary), 1970, " Is Semantics Possible? ", in H. KIEFER \& M. MunITz, eds, Languages, Belief and Metaphysics, New York, State University of New York.

Putnam (Hilary), 1975, "The Meaning of 'Meaning' ", in K. Gunderson, ed., Language, Mind and Knowledge, Minneapolis, Minnesota, University of Minnesota Press.

Quine (Willard Van Orman), 1960, Word and Object, Cambridge, MA, MIT Press.

QUINE (Willard Van Orman), 1974, The Roots of Reference (The Paul Carus Lectures), La Salle, Ill., Open Court.

RAPPAPORT (Malka) \& LEVIN (Beth), 1988, "What to Do with Theta-Roles ", in W. Wilkins, ed., Thematic Relations, New York, Academic Press. 
RiemsDiJk (Henk Van) \& Williams (Edwin), 1986, Introduction to the Theory of Grammar, Cambridge, MA, MIT Press.

Roeper (Thomas) \& Williams (Edwin), eds, 1987, Parameter Setting, Dordrecht, Netherlands, D. Reidel.

SAlASOO (A.) \& Pisoni (David B.), 1985, "Interaction of Knowledge Sources in Spoken Word Identification ", Journal of Memory and Language, 24, p. $210-$ 231.

Segal (Gabriel) \& Sober (Elliott), 1989, "The Causal Efficacy of Content ", manuscrit non publié, Madison, University of Wisconsin, Department of Philosophy.

SMrTH (Edward E.) \& MEdIN (Douglas L.), 1981, Categories and Concepts, Cambridge, MA, Harvard University Press.

SPelke (Elisabeth S.), 1985, « Perception of Unity, Persistence and Identity: 'Thoughts on Infants' Conception of Objects ", in J. MEHLER \& R. Fox, eds, Neonate Cognition : Beyond the Blooming Buzzing Confusion, Hillsdale, NJ, Lawrence Erlbaum.

SPELKE (Elisabeth S.), 1988, «Where Perceiving Ends and Thinking Begins: The Apprehension of Objects in Infancy », in A. Yonas, ed., Perceptual Development in Infancy, Minneapolis, MI, University of Minnesota Press.

TALMY (Leonard), 1985, " Lexicalization Patterns ", in T. SHOPEN, ed., Language Typology and Syntactic Description, Cambridge, U.K., Cambridge University Press.

Tanenhaus (Michael K.), Garnsey (Susan M.) \& Boland (Julie), 1989, "Combinatory Lexical Information and Language Comprehension ", in G. Altman, ed., Cognitive Models of Speech Processing : Psycholinguistic and Computational Perspectives, Cambridge, MA, MIT Press.

TEnny (Carol), ed., 1988 a, Studies in Generative Approaches to Aspect, Lexicon Project Working Papers, $n^{\circ}$ 24, Cambridge, MA, MIT Center for Cognitive Science.

Tenny (Carol), 1988 b, "The Aspectual Interface Hypothesis ", in C. Tenny, ed., 1988 a, p. $1-18$. 